\title{
CAPACITÉ DES ENSEMBLES PRODUITS
}

\author{
MAKOTO OHTSUKA
}

\section{Introduction}

Si $X$ est un ensemble mesurable au sens de Lebesgue dans un espace euclidien $E_{n}$ et $Y$ est un ensemble mesurable au sens de Lebesgue dans un autre espace euclidien $E_{m}$, alors $X \times Y$ est mesurable dans $E_{n+m}=E_{n} \times E_{m}$ et sa mesure est égale au produit de la mesure de $X$ et celle de $Y$. Mais il n'existe pas de telle relation simple ni pour la capacité d'ordre général ni pour la mesure de Hausdorff de dimension générale. Dans le présent mémoire nous essayons d'évaluer la capacité des ensembles produits au moyen de la capacité ou de la mesure de Hausdorff des ensembles composants.

Le premier paragraphe est consacré aux préliminaires. Il s'agira d'un espace métrique localement compact et des noyaux $\log 1 / \rho, 1 / \rho^{x}$ et $\log ^{+} 1 / \rho$, où $\rho$ est la distance et, en général, $f^{+}$signifie $\max (f, 0)$. L'étude des potentiels pour des noyaux plus généraux dans un espace localement compact est courante dans la théorie du potentiel, mais dans le présent mémoire nous nous limitons strictement à ce qui sera nécessaire dans les paragraphes suivants; une discussion sera faite d'un point de vue général dans un mémoire en préparation.

Il est bien connu que la dimension capacitaire est égale à la dimension hausdorffienne dans l'espace euclidien. Ainsi la connaissance de la valeur de mesure de Hausdorff est utile en déterminant la valeur de capacité. Il y a d'assez nombreuses recherches sur la mesure de Hausdorff des ensembles produits. Nous donnerons dans 1.6 un exposé rapide des résultats connus sur ce sujet. En particulier, nous citons ici un phénomène pathologique trouvé par Besicovitch-Moran [4] disant que, étant donnés $\alpha$ et $\beta, 0<\alpha, \beta \leqq 1$, il existe un ensemble fermé $X$ de dimension $\alpha$ sur l'axe des $x$ et un ensemble fermé $Y$ de dimension $\beta$ sur l'axe des $y$ tels que la dimension de $X \times Y$ soit égale à $\min (1+\alpha, 1+\beta)$.

Received May 22, 1957 . 
L'objet des $\$ \S 2-3^{11}$ est d'évaluer la capacité des ensembles produits. Le seule travail qui a explicitement traité ce problème est [10] par Deny-Lelong qui montre qu'un ensemble cylindrique dans l'espace euclidien $E_{n}(n \geq 3)$ est de capacité extérieure d'ordre $n-2$ nulle si et seulement si l'ensemble à base est de capacité extérieure d'ordre $n-3$ (ou capacité logarithmique extérieure si $n=3$ ) nulle. La capacité des ensembles produits est minorée dans $\S 2$ et majorée dans $\S 3$, au moyen de la capacité ou de la mesure de Hausdorff des ensembles composants.

Soient $X$ et $Y$ des ensembles compacts. Le résultat de Besicovitch-Moran mentionné ci-dessus suggère qu'il n'existe pas de limitation supérieure pour la dimension de $X \times Y$ si on ne tient pas compte des dimensions des deux espaces dans lesquels $X$ et $Y$ sont respectivement placés. En effet, on montrera dans $\$ 4$ que, si $X \subset E_{n}$ et $Y \subset E_{m}$, on a

$$
\operatorname{dim}(X)+\operatorname{dim}(Y) \leqq \operatorname{dim}(X \times Y) \leqq \min (n+\operatorname{dim}(Y), m+\operatorname{dim}(X))
$$

et que, étant donnés $\alpha, 0<\alpha \leqq n$, et $\beta, 0<\beta \leqq m$, on peut trouver un compact $X \subset E_{n}$ de dimension $\alpha$ et un compact $Y \subset E_{m}$ de dimension $\beta$ tels que $\operatorname{dim}(X \times Y)$ $=\min (n+\beta, m+\alpha)$. Besicovitch et Moran ont évalué la mesure de Hausdorff mais nous compterons la capacité d'un ensemble de Cantor généralisé.

Ce travail a son origine dans le problème de trouver des critères pour qu'un ensemble soit effilé en un point. De fait nos résultats sur l'évaluation de la capacité des ensembles produits peuvent s'appliquer au problème d'effilement, mais on prendra une autre occasion pour cette application; il faudra étendre une condition de Wiener-Brelot pour l'effilement.

\section{§ 1. Potentiel, capacité et dimension}

1. 1. L'espace $\Omega$ dont il s'agira sera un espace métrique localement compact, et les noyaux seront les fonctions particulières de la distance $\rho(P, Q)$ :

$$
\mathscr{D}(P, Q)=\log \frac{1}{\rho(P, Q)} \quad \text { et } \quad \frac{1}{\rho^{\alpha}(P, Q)} \quad(0<\alpha<\infty) .
$$

Les potentiels pour ces noyaux, engendrés par une mesure de Radón positive finie $/$ à support compact, sont définis par

1) Un résumó de résultats partiels a été donné dans Bull. Amer. Math. Soc., 59 (1953), pp. 453-454. 


$$
\int_{\Omega} D(P, Q) d \ell(Q)
$$

et notés $U_{0}^{\prime \prime}(P)$ et $U_{\alpha}^{\perp}(P),{ }^{2 !}$ et ils sont appelés le 0-potentiel ou le potentiel logarithmique et l' $\alpha$-potentiel respectivement; une mesure de Radon " sera toujours positive finie à support compact dans ce mémoire et son support sera noté $S_{\mu}$.

On définit l'énergie $(\mu, \mu)$ par

$$
\iint \mathscr{D}(P, Q) d \mu(P) d \mu(Q)
$$

et désigne par (5. l'ensemble des mesures dont l'énergie est finie. Nous désignons par $\tilde{f}_{x}$ l'ensemble des mesures de masse totale unité dont le support est contenu dans un ensemble $X$.

Posons pour un compact $K \subset \Omega$ non vide

$$
W(K)=\inf _{\mu \in \tilde{Y}_{K}}(\mu, \mu) .
$$

A cause d'un théorème de choix (voir [j], par exemple), il existe au moins une mesure $\mu_{0} \in \tilde{f}_{K}$ qui rend l'énergie minimum. De plus, par le raisonnement habituel on peut démontrer la

Proposition 1. On $a$

et

$$
U_{x}^{j_{0}}(P) \equiv \mathbb{W}(K) \quad \text { partout sur } S_{\mu_{0}}
$$

$$
U_{\alpha}^{\prime o}(P) \rightleftharpoons W(K)
$$

sur $K$ sauf sur un $F_{\sigma}$ dont la masse est nulle pour toute $\mu \in(\dot{E}$.

Pour la démonstration voir $[17 ; 19]$.

1.2. On dit qu'un noyau satisfait au principe du maximum c-dilaté $(c \geqslant 0)^{3}$ si pour toute $\mu$ on a

$$
\sup _{\Gamma \in S 2} U_{\alpha}^{\mu}(P) \leqq c \sup _{P \subseteq N_{\mu}} U_{\alpha}^{\prime 2}(P)
$$

On va démontrer la

Proposition 2. Le noyau $\rho^{-x}(P, Q)(\alpha>0)$ satisfait au principe du maxi-

2) En outre, on considérera dans 1.7 le noyau $\log ^{+} 1, p=\max (\log 1, \rho, 0)$ et désignera le potentiel pour ce noyau par $\dot{U}_{4}^{\mu}(P)$.

3. Une terminologie dans [8]. 
mum $2^{\alpha}$-dilaté. ${ }^{4)} \quad$ Pour le noyau $\log 1 / \rho(P, Q)$, on a

$$
\sup _{P \in \Omega} U_{0}^{\mu}(P) \leqq \sup _{P \in S_{\mu}} U_{0}^{\mu}(P)+\mu(\Omega) \log 2 .
$$

En effet, pour $P \notin S_{\mu}$ on désigne par $P_{1} \in S_{\mu}$ un point tel que $\rho\left(P, P_{1}\right)$ $=\inf _{Q \in s_{\mu}} \rho(P, Q)$. Alors

$$
\rho\left(P_{1}, Q\right) \leqq \rho\left(P, P_{1}\right)+\rho(P, Q) \leqq 2 \rho(P, Q),
$$

et donc

$$
U_{\alpha}^{\mu}(P)=\int_{\Omega} \frac{1}{\rho^{\alpha}(P, Q)} d \mu(Q) \leqq 2^{\alpha} \int_{Q} \frac{1}{\rho^{\alpha}\left(P_{1}, Q\right)} d_{\mu}(Q)=2^{\alpha} U_{\alpha}^{\mu}\left(P_{1}\right)
$$

ou

$$
U_{0}^{\mu}(P)=\int_{\Omega} \log \frac{1}{\rho(P, Q)} d \mu(Q) \leqq U_{0}^{\mu}\left(P_{1}\right)+\mu(\Omega) \log 2,
$$

d'où la proposition.

En vertu de cette propriété de nos noyaux, les potentiels font beaucoup de caractères communs avec le potentiel newtonien. Cependant, nous ne nous intéressons dans la suite qu'aux propriétés dont nous aurons besoin dans les pragraphes 2 et 3 .

1. 3. Nous définirons deux espèces de capacité. D'abord nous poserons pour un ensemble compact $K$

$$
V_{\mu}^{(\alpha) *}=\sup _{P \in S_{\mu}} U_{\alpha}^{\mu}(P) \quad \text { et } \quad V_{\alpha}^{*}(K)=\inf _{\mu \in \mathscr{F}_{K}} V_{\mu}^{(\alpha) *} \quad(\alpha \geqslant 0)
$$

si $K$ n'est pas vide, et $V_{a}^{*}(\phi)=\infty$. D'après la proposition 1 , on a $V_{a}^{*}(K) \leqq W(K)$. D'autre part, on trouve $V_{a}^{*}(K) \geqslant W(K)$, car pour toute $\mu \in \mathfrak{F}_{K}$

$$
W(K) \leqq \int_{\Omega} U_{\alpha}^{\mu}(P) d \mu(P) \leqq \sup _{P \in s_{\mu}} U_{\alpha}^{\mu}(P) .
$$

Ainsi on a la

Proposition 3. $\quad W(K)=V_{\alpha}^{*}(K)$.

On pose pour un ensemble quelconque $X$

$$
\left.C_{i}^{(\alpha) *}(X)=\exp \left[-\inf _{K \subset X} V_{0}^{*}(K)\right] \quad(\alpha=0) \quad \text { ou } \quad \inf _{K \subset X} V_{\alpha}^{*}(K)\right\}^{-1} \quad(\alpha>0),
$$

où $K$ est un ensemble compact, et l'on pose

$$
C_{e}^{(\alpha) *}(X)=\sup _{Y \subset X} \inf _{G \supset Y} C_{i}^{(\alpha) *}(G), \quad(\alpha \geqslant 0),
$$

4) C'est un cas particulier du critère 3 du $n^{0} 2$ de [8]. 
où $Y$ est un ensemble relativement compact dans $\Omega$ et $G$ est un ensemble ouvert. Les deux fonctions des ensembles seront appelées l'a-capacité intérieure restreinte et l' $\alpha$-capacité extérieure restreinte de $X$ respectivement. Ici on ne définit pas $C_{e}^{(\alpha) *}(X)$ par $\inf _{G \supset X} C_{i}^{(\alpha) *}(G)$; on peut démontrer qu'un ensemble est $\alpha$-polaire si et seulement si son $\alpha$-capacité extérieure (restreinte) est nulle; voir 1.4 .

Pour définir une autre espèce de capacité nous posons

$$
V_{\mu}^{(\alpha)}=\sup _{P \in \Omega} U_{\alpha}^{\mu}(P) \quad \text { et } \quad V_{\alpha}(K)=\inf _{\mu \in \widetilde{\mho}_{K}} V_{\mu}^{(\alpha)}
$$

si $K \neq \phi$ et $V_{\alpha}(\phi)=\infty$ si $K=\phi$, et définissons l' $\alpha$-capacité intérieure par

$$
C_{i}^{(\alpha)}(X)=\exp \left[-\inf _{K \subset X} V_{0}(K)\right] \quad(\alpha=0) \quad \text { ou } \quad\left\{\inf _{K \subset X} V_{\alpha}(K)\right\}^{-1} \quad(\alpha>0)
$$

et l' $\alpha$-capacité extérieure par

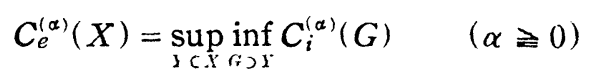

comme plus haut.

Les $\alpha$-capacités (restreintes resp.) sont autrement appelées les capacités ( restreintes resp.) d'ordre $\alpha$. Elles sont des fonctions croissantes des ensembles, et

$$
\begin{aligned}
& C_{i}^{(\alpha) *}(X) \leqq C_{e}^{(\alpha) *}(X), \quad C_{i}^{(\alpha)}(X) \leqq C_{e}^{(\alpha)}(X), \\
& C_{i}^{(\alpha)}(X) \leqq C_{i}^{(\alpha) *}(X), \quad C_{e}^{(\alpha)}(X) \leqq C_{e}^{(\alpha) *}(X) \quad(\alpha \geqslant 0) \text {. }
\end{aligned}
$$

On a aussi la

Proposition 4.5)

$$
\begin{aligned}
& \left\{C_{i}^{(\alpha) *}(X)\right\}^{1 / \alpha} \geqq\left\{C_{i}^{(\beta) *}(X)\right\}^{1 / 3}, \quad\left\{C_{i}^{(\alpha)}(X)\right\}^{1 / \alpha} \geqq\left\{C_{i}^{(\beta)}(X)\right\}^{1 / 3} \quad \text { pour } 0<\alpha<\beta, \\
& \text { et } \\
& C_{i}^{(0) *}(X) \geqq\left\{C_{i}^{(\alpha) *}(X)\right\}^{1 / \alpha}, \quad C_{i}^{(0)}(X) \geqq\left\{C_{i}^{(\alpha)}(X)\right\}^{1 / \alpha} \quad \text { pour } \quad \alpha>0 ;
\end{aligned}
$$

les relations analogues sont vraies aussi pour $C_{e}^{(\alpha) *}(X)$ et $C_{e}^{(\alpha)}(X)(\alpha \geqslant 0)$.

D'après la proposition 2 , on a $C_{i}^{(0) *}(X) \leqq 2 C_{i}^{(0)}(X)$ et $C_{e}^{(0) *}(X) \leqq 2 C_{e}^{(0)}(X)$, et pour $\alpha>0$ on a $C_{i}^{(\alpha) *}(X) \leqq 2^{\alpha} C_{i}^{(\alpha)}(X)$ et $C_{e}^{(\alpha) *}(X) \leqq 2^{\alpha} C_{e}^{(\alpha)}(X)$. Donc on obtient la

5) Voir, par exemple, p. 52, prob. 71 de Pólya-Szegö: Aufgaben und Lehrsätze aus der Analysis, I, Berlin (1925), ou les théorèmes 184 et 192 de Hardy-Littlewood-Pólya: Inequalities, Cambrige (1952). 
Proposition 5. Pour tout $X, C_{i}^{(\alpha) *}(X)=0\left(C_{e}^{(\alpha) *}(X)=0\right.$ resp. $)$ si et seulement si $C_{i}^{(\alpha)}(X)=0\left(C_{e}^{(\alpha)}(X)=0\right.$ resp. $)$.

Par conséquent, tout critère pour qu'une capacité restreinte soit nulle est valable aussi pour la capacité correspondante et l'inverse est vrai; on ne donnera donc des critères que pour $C_{i}$ et $C_{e}$.

On peut établir sans aucune difficulté la

Proposition 6. Soient $\left\{B_{n}\right\}(n=1,2, \ldots)$ des ensembles boréliens. Les égalités $C_{i}^{(\alpha)}\left(B_{n}\right)=0(\alpha \geq 0)$ entraînent $C_{i}^{(\alpha)}\left(\cup B_{n}\right)=0$. Il en est même de $C_{e}^{(\alpha)}$.

Le détail de la démonstration sera publié ultérieurement.

On dira que $X$ est $\alpha$-capacitable ( $\alpha$-capacitable au sens restreint resp.) si

$$
C_{i}^{(\alpha)}(X)=C_{e}^{(\alpha)}(X) \quad\left(C_{i}^{(\alpha) *}(X)=C_{e}^{(\alpha) *}(X) \text { resp. }\right),
$$

et on écrira la valeur commune $C^{(\alpha)}(X)\left(C^{(\alpha) *}(X)\right.$ resp.). Evidemment tout ensemble ouvert est $\alpha$-capacitable aux deux sens. On peut démontrer aussi que tout ensemble compact est $\alpha$-capacitable $(\alpha \geqslant 0)$ aux deux sens.

Proposition 7.

$$
C_{i}^{(\alpha)}(K)=C_{e}^{(\alpha)}(K) \quad \text { et } \quad C_{i}^{(\alpha) *}(K)=C_{e}^{(\alpha) *}(K) \quad(\alpha \geq 0) .
$$

En effet, on prend une suite d'ensembles ouverts $\left\{G_{n}\right\}$ telle que $G_{n} \searrow K$ et que l'adhérence $G_{n}^{a}$ de $G_{n}$ soit compacte et contenue dans $G_{n-1}(n=1,2, \ldots)$. Soit $\mu_{n} \in \mathfrak{F}_{G_{n}}{ }^{a}$ une répartition qui rend $\left(\mu_{n}, \mu_{n}\right)=\inf _{\mu \in \mathfrak{F}_{G_{k}}{ }^{a}}(\mu, \mu)$. On peut extraire une suite partielle $\left\{\mu_{n_{i}}\right\}$, grâce à un théorème de choix, qui converge vaguement vers une mesure $\mu_{0} \in \mathfrak{F}_{K}$. Alors on a $\lim _{k \rightarrow \infty}\left(\mu_{n_{k}}, \mu_{n_{k}}\right) \geqslant\left(\mu_{0}, \mu_{0}\right)$. D'autre part $\left(\mu_{i}, \mu_{0}\right) \geqq\left(\mu_{n_{k}}, \mu_{n_{k}}\right)$ car $\mu_{0} \in \widetilde{F}_{G_{n}}{ }^{a}$ pour tout $n$, et donc $\lim _{k \rightarrow \infty}\left(\mu_{n_{k}}, \mu_{n_{k}}\right)$ $=\left(\mu_{0}, \mu_{0}\right)$. Comme $\left(\mu^{\prime}, \mu^{\prime}\right) \gtrsim\left(\mu_{n_{k}}, \mu_{n_{k}}\right)$ si $\mu^{\prime} \in \mathfrak{F}_{K}$, on en conclut que $\left(\mu_{0}, \mu_{0}\right)$ $=\inf (\mu, \mu)=W(K)$. Il résulte d'après la proposition 3 que $\mu \in \mathfrak{F} \mathrm{K}$

$$
\begin{aligned}
\left(\mu_{0}, \mu_{0}\right)^{-1}=V_{\alpha}^{*}(K)^{-1} & =C_{i}^{(\alpha) *}(K) \leqq C_{e_{i}}^{(\alpha) *}(K) \leqq C^{(\alpha)}\left(G_{n_{k}}\right) \leqq C_{i}^{(\alpha) *}\left(G_{n_{k}}^{a}\right) \\
& =V^{*}\left(G_{n_{k}}^{a}\right)^{-1}=\left(\mu_{n_{n_{k}}}, \mu_{n_{k}}\right)^{-1} \quad \text { pour } \quad \alpha>0
\end{aligned}
$$

et

$$
e^{-\left(\mu_{0}, \mu_{0}\right)}=C_{i}^{(0) *}(K) \leqq C_{e}^{(0) *}(K) \leqq C^{(0) *}\left(G_{n_{k}}\right) \leqq e^{-\left(\mu_{n_{k}}, \mu_{n_{k}}\right)},
$$

d'où l'égalité

$$
C_{i}^{(\alpha) *}(K)=C_{e}^{(\alpha) *}(K) \quad \text { pour } \quad \alpha \geq 0
$$


Pour démontrer $C_{i}^{(\alpha)}(K)=C_{e}^{(\alpha)}(K)$, on prend $\nu_{n} \in \mathfrak{\mho}_{G_{n}} a$ telle que $V_{\nu_{n}}^{(\alpha)}<V_{\alpha}\left(G_{n}^{a}\right)$ $+\frac{1}{n}$ et on choisit $\left\{\nu_{n_{k}}\right\}$ qui converge vaguement vers $\nu_{0} \in \widetilde{F}_{K}$. Alors $\lim _{k \rightarrow \infty} U^{\nu_{n_{k}}}(P)$ $\geq U^{\nu_{0}}(P)$ dans $\Omega$ et donc $\lim _{k \rightarrow \infty} V_{\nu_{n}}^{(\alpha)} \geq V_{\nu_{0}}^{(\alpha)}$, d'où $\lim _{n \rightarrow \infty} V_{\alpha}\left(G_{n}^{a}\right) \geq V_{\alpha}(K)$. Par conséquent $C_{e}^{(\alpha)}(K) \leqq C_{i}^{(\alpha)}(K)$. L'inverse est évident et on en déduit l'égalité $C_{i}^{(\alpha)}(K)$ $=C_{e}^{(\alpha)}(K)$.

Si les ensembles sont dans un espace euclidien $E_{n}$, nous avons la

Proposition 8. Tout ensemble analytique relativement compact dans $E_{n}$ est $\alpha$-capacitable $(\alpha \geqq 0)$ au sens restreint.

Pour la démonstration, nous renvoyons à [1] (voir aussi [7]).

1. 4. Un ensemble $X$ relativement compact dans $\Omega$ sera appelé $\alpha$-polaire $(\alpha \geqslant 0)$ s'il existe un potentiel $U_{\alpha}^{\mu}(P)$ qui est égal à $\infty$ en chaque point de $X$. Un ensemble quelconque sera appelé $\alpha$-polaire si tout sous-ensemble relativement compact dans $\Omega$ est $\alpha$-polaire.

Proposition 9. L'a-capacité extérieure d'un ensemble $\alpha$-polaire $X$ est nulle.

On peut supposer que $X$ est relativement compact dans $\Omega$. Supposons qu'il existe un potentiel $U_{\alpha}^{\prime \prime}(P)$ qui est égal à $\infty$ en chaque point de $X$. Comme $U_{\alpha}^{\mu}(P)$ est semi-continu inférieurement, l'ensemble $G_{n}=\left\{P ; U_{\alpha}^{\alpha}(P)>n\right\}$ est un ensemble ouvert contenant $X$. Soit $\nu$ une mesure quelconque de $\tilde{F}_{i_{n}}$. Alors de la relation

$$
n<\int_{\Omega} U_{\alpha}^{\mu}(P) d \nu(P)=\int_{\Omega} U_{\alpha}^{\nu}(P) d \mu(P)
$$

on tire l'inégalité $\sup _{P \in \Omega} U_{\alpha}^{\nu}(P)>n$, d'où $C_{i}^{(\alpha)}\left(G_{n}\right) \leqq 1 / n$ et donc $C_{e}^{(\alpha)}(X)=0$.

L'inverse de cette proposition est vrai mais ne sera pas démontré ici; on n'aura pas besoin de ce fait dans ce mémoire. Cependant, la proposition suivante nous fournira des ensembles $\alpha$-polaires; elle est une généralisation du théorème dit d'Evans ou d'Evans-Selberg.

Proposition 10. Tout ensemble compact $K d^{\prime} \alpha$-capacité (restreinte) nulle porte une mesure $\mu$ de masse-unité telle que $U_{\alpha}^{\mu}(P)=\infty$ en chaque point de $K$.

La démonstration peut être faite comme d'habitude. Certainement $K$ est alors $\alpha$-polaire; une réunion dénombrable d'ensembles compacts d' $\alpha$-capacité (restreinte) nulle est aussi $\alpha$-polaire. Des propositions 1 et 3 on tire la 
Proposition 11. Soit $K$ un ensemble compact. Pour tout $\varepsilon>0, K$ porte une mesure $\mu$ de masse totale unité telle que

$$
U_{\alpha}^{\mu}(P) \geq \frac{V_{\alpha}^{*}(K)}{1+\varepsilon} \quad \text { partout sur } K .
$$

1.5. La dimension capacitaire intérieure (extérieure resp.) d'un ensemble quelconque $X$ dans un espace métrique localement compact est définie par

$$
\inf \left\{\alpha ; C_{i}^{(\alpha)}(X)=0 \quad\left(C_{e}^{(\alpha)}(X)=0 \text { resp. }\right)\right\}
$$

et notée $\operatorname{dim}_{c i}(X) \quad\left(\operatorname{dim}_{c e}(X)\right.$ resp.); cette définition se fonde sur la proposition 4. A cause de la proposition 5 , il ne faut pas définir la dimension par rapport aux capacités restreintes.

Or nous définirons la mesure de Hausdorff d'un ensemble quelconque $X$ dans l'espace euclidien $E_{n}$ de dimensions. $n$ Considérons

$$
m_{0}^{(\varepsilon)}(X)=\inf _{\left\{H_{k}\right\}} \sum_{k} \frac{1}{\log \frac{1}{\operatorname{diam} H_{k}}} \quad \text { si } \alpha=0
$$

ou

$$
m_{\alpha}^{(\varepsilon)}(X)=\inf _{\left(H_{k}\right)} \sum_{k}\left(\operatorname{diam} H_{k}\right)^{\alpha} \quad \text { si } \alpha>0,
$$

où $\left\{H_{k}\right\}$ est un recouvrement dénombrable de $X$ tel que tout diam $H_{k}$ soit $<\varepsilon$. Nous appellerons $\lim _{\varepsilon \rightarrow 0} m_{\alpha}^{(\varepsilon)}(X)$ la mesure de Hausdorff de dimension $\alpha$ et la noterons $m_{\alpha}(X)(\alpha \geq 0)$. La mesure intérieure $m_{\alpha}(X)$ est définie par sup $m_{\alpha}(K)$ par rapport aux ensembles compacts $K$ contenus dans $X$. Si un ensemble $X$ est $m_{\alpha}$-mesurable et $m_{\alpha}(X)<\infty$, alors $m_{\alpha}(X)=\underline{m}_{\alpha}(X)$.

Nous avons les relations suivantes entre les capacités et les mesures de Hausdorff.

Proposition 12. Soit $X \subset E_{n}$. Si $m_{\alpha}(X)>0$ alors $C_{e}^{(\beta)}(X)>0$ pour tout $\beta, 0 \leqq \beta<\alpha$, et si $m_{a}(X)>0$ alors $C_{i}^{(\beta)}(X)>0$ pour tout $\beta, 0 \leqq \beta<\alpha$.

On trouve une démonstration du premier énoncé dans [6] et [20]. Si $m_{\alpha}(X)>0$ il existe un compact $K \subset X$ tel que $m_{\alpha}(K)>0$. D'après le premier énoncé $C_{e}^{(\beta)}(K)>0$ pour tout $\beta, 0 \leqq \beta<\alpha$. Par la proposition 7 on a $C_{i}^{(\beta)}(X)$ $\geq C_{i}^{(\beta)}(K)=C_{e}^{(\beta)}(K)>0$.

Inversement on a la

Proposition 13. Soit $X \subset E_{n}$. Si $C_{e}^{(\alpha)}(x)>0(\alpha \geqslant 0)$ alors $m_{\alpha}(X)=\infty$, et si $C_{i}^{(\alpha)}(X)>0$ alors $\underline{m}_{\alpha}(X)=\infty$. 
Une démonstration du deuxiẻme énoncé se trouve sous une forme plus générale dans [18]. Ensuite supposons que $C_{e}^{(\alpha)}(X)>0$; soit $X_{1} \subset X$ relativement compact tel que $C_{e}^{(\alpha)}\left(X_{1}\right)>0$. Puisque $m_{\alpha}(X)$ est une mesure extérieure régulière, il existe un ensemble relativement compact $G_{\delta} \supset X_{1}$ tel que $m_{\alpha}\left(G_{\delta}\right)$ $=m_{\alpha}\left(X_{1}\right)$. Comme $G_{\delta}$ est capacitable au sens restreint d'après la proposition $8, C_{i}^{(\alpha)}\left(G_{\delta}\right)=C_{e}^{(\alpha)}\left(G_{\delta}\right)>0$ et, en vertu du deuxième énoncé, $m_{\alpha}\left(X_{1}\right)=m_{\alpha}\left(G_{\delta}\right)=\infty$.

On définit la dimension hausdorffienne $\operatorname{dim}_{h}(X)$ d'un ensemble $X$ dans un espace euclidien par

$$
\inf \left\{\alpha ; m_{\alpha}(X)=0\right\} \text {, }
$$

et la dimension hausdorffienne intérieure $\operatorname{dim}_{h i}(X)$ par

$$
\inf \left\{\alpha ; \underline{m}_{\alpha}(X)=0\right\} \text {. }
$$

On tire des propositions 12 et 13 la

Proposition 14. $\operatorname{dim}_{c e}(X)=\operatorname{dim}_{h}(X) \geqslant \operatorname{dim}_{c i}(X)=\operatorname{dim}_{h i}(X)$ pour tout $X$ dans un espace eucldien. Si $\underline{m}_{a}(X)>0$ pour tout $\alpha>0$ tel que $m_{\alpha}(X)>0{ }^{6}{ }^{1}$ toutes les dimensions sont égales.

Les abréviations $\operatorname{dim}_{c}(X)$ et $\operatorname{dim}(X)$ auront les significations évidentes.

1. 6. Les propositions 12 et 13 montrent que la connaissance de valeurs de la mesure (ou mesure intéricure resp.) de Hausdorff des ensembles produits est utile en étude de la capacité extérieure (intérieure resp.) des enstmin. produits. Il y a d'assez nombreux travaux sur celle-là; nous citons ici Hausdorff [16], Randolph [25], Besicovitch et Moran [4], Moran [22; 23], Eggleston [11; 12], Freilich [14; 15], Federer [13] et Marstrand [21].

Nous allons faire un exposé sur certains de leurs résultats. Soit $Z$ un ensemble dans le plan $x+i y$ et soit $X$ un ensemble sur l'axe des $x$. Désignon: par $Z_{x}$ l'intersection de $Z$ avec la droite de l'abscisse $x$. Marstrand [21] a montic que si $p$ est un nombre positif tel que pour tout $x \in X$ on ait $m_{\beta}\left(Z_{x}\right)>p$, alors il existe une constante absolue $c>0$ telle que l'inégalité

$$
m_{\alpha+\beta}(Z) \gtrsim c p m_{\alpha}(X)
$$

soit vraie pour tout $\alpha>0$. De cette relation on tire immédiatement

6) Voir la fin de 1.6. Évidemment $m_{\alpha}(X)>0$ entralne $m_{\beta}(X)>0$ pour tout $\beta, 0 \leqq \beta<\alpha$. 


$$
m_{\alpha+\beta}(X \times Y) \geq c m_{\alpha}(X) m_{\beta}(Y)
$$

et

$$
\operatorname{dim}_{h}(X \times Y) \geq \operatorname{dim}_{h}(X)+\operatorname{dim}_{h}(Y) ;
$$

une généralisation de cette relation sera donnée dans le corollaire 3 du théorème 2 .

L'inégalité dans l'autre sens n'est pas vraie en général. Les résultats suivants ont été obtenus par Besicovitch et Moran [4]. Si $X$ est un ensemble $m_{\alpha}$-mesurable sur l'axe des $x$ et sa $\operatorname{dim}_{h}$ est égale à $\alpha$ et si $Y$ est un ensemble $m_{\beta}$-mesurable sur l'axe des $y$ et sa $\operatorname{dim}_{h}$ est égale à $\beta$, alors

$$
\operatorname{dim}_{h}(X \times Y) \leqq 1+\min (\alpha, \beta)
$$

et, pour $\alpha$ et $\beta, 0<\alpha, \beta \leqq 1$, quelconques, on trouve des ensembles fermés $X$ et $Y$ qui réalisent l'égalité. $\mathrm{Si}$, de plus, $0<m_{\alpha}(X)<\infty$ et $0<m_{\beta}(Y)<\infty$ et si la densité inférieure de $Y$ par rapport à la mesure $m_{\beta}$ est positive en tout point de $Y$, alors

$$
\operatorname{dim}_{h}(X \times Y)=\operatorname{dim}_{h}(X)+\operatorname{dim}_{h}(Y)=\alpha+\beta .
$$

Si $m_{1}(X)<\infty$, on a

$$
m_{1}(X) m_{\beta}(Y) \leqq m_{1+\beta}(X \times Y) \leqq 2^{(1+\beta) / 2} m_{1}(X) m_{\beta}(Y) .
$$

Même si $X$ est un segment et $Y$ est un ensemble plan $m_{1}$-mesurable, il $y$ a un cas où

$$
m_{1}(X) m_{1}(Y)<m_{2}(X \times Y) ;
$$

la question pour l'existence de tel exemple avait été posée par Randolph [25].

Freilich [14] a rétabli (1.5) et Moran [23] et Federer [13] ont obtenu certaines inégalités pareilles à (1.4).

Un ensemble $X$ est appelé un $\alpha$-ensemble s'il contient un ensemble compact de $m_{\alpha}$-mesure positive finie. La question à savoir quand un ensemble $m_{\alpha^{-}}$ mesurable $X$ de $m_{\alpha}(X)=\infty$ est un $\alpha$-ensemble a été étudiée par Besicovitch $[2 ; 3]$ et Davies [9]. Ils ont montré que c'est le cas de tout ensemble analytique mais non pas toujours l'est. Donc $\underline{m}_{\alpha}(X)>0$ si $X$ est analytique et $m_{\propto}(X)>0$.

1. 7. Pour l'utiliser dans certaines évaluations de capacité, on considérera une autre espèce de capacité logarithmique. On désigne par $\stackrel{U}{0}_{0}^{\mu}(P)$ le potentiel 
pour le noyau $\log ^{\dagger} 1 / \rho(P, Q)$, et par $\stackrel{+}{V}_{0}^{*}(K)$ et $\stackrel{\perp}{V}_{0}(K)$ les quantités correspondantes à $V_{0}^{*}(K)$ et $V_{0}(K)$ respectivement. Nous définissons $\stackrel{\perp}{C}_{i}^{(0) *}(X)$ et $\stackrel{+}{C}_{i}^{(0)}(X)$ encore par $\exp \left[-\inf \stackrel{+}{V}_{0}^{*}(K)\right]$ et $\exp \left[-\inf \vec{V}_{0}(K)\right]$ respectivement; la signification de $\stackrel{+}{C}_{e}^{(0) *}(X)$ et $\stackrel{+}{C}_{e}^{(0)}(X)$ sera manifeste.

La proposition 1 est vraie aussi pour $\dot{U}_{0}^{\prime \prime}(P)$ et on indiquera ce fait comme la proposition $1^{\prime}$.

On a évidemment

$$
\begin{aligned}
& \stackrel{+}{C}_{i}^{(0) *}(X) \leqq C_{i}^{(0) *}(X), \stackrel{+}{C}_{i}^{(0)}(X) \leqq C_{i}^{(0)}(X), \\
& \stackrel{+}{C}_{c}^{(0) *}(X) \leqq C_{e}^{(0) *}(X), \stackrel{\stackrel{C}{C}}{e}^{(0)}(X) \leqq C_{e}^{(0)}(X)
\end{aligned}
$$

et

$$
\begin{aligned}
& \stackrel{+}{C}_{i}^{(0) *}(X) \leqq \stackrel{+}{C}_{e}^{(0) *}(X), \stackrel{+}{C}_{i}^{(0)}(X) \leqq \dot{C}_{e}^{(0)}(X), \\
& \stackrel{+}{C}_{i}^{(0)}(X) \leqq \dot{C}_{i}^{(0 ; *}(X), \dot{C}_{e}^{(0)}(X) \leqq \dot{C}_{e}^{(0) *}(X) .
\end{aligned}
$$

Un analogue de la proposition 5 est vrai pour $\stackrel{t}{C}$. On a aussi la

Proposition 15. $\stackrel{\grave{C}}{\boldsymbol{C}}_{i}^{(0)}(X)=0\left(\stackrel{\grave{C}}{\mathrm{C}}_{e}^{(0)}(X)=0\right.$ resp. $)$ si et seulement si $C_{i}^{(0)}(X)$ $=0\left(C_{e}^{(0)}(X)=0\right.$ resp. $)$.

Supposons que $C_{i}^{(0)}(K)>0$ pour un compact $K \subset X$. Nous désignons le diamètre de $K$ par $d<\infty$. Si la distance de $P$ à $S_{\imath}$ est $\geqq 1$, on a

$$
\stackrel{U}{0}_{0}^{\prime}(P)=\int \log _{\rho}^{+1} d \mu=0
$$

pour $\mu \in \tilde{F}_{K}$ quelconque, et si la distance est $<1$, on a

$$
\dot{U}_{0}^{\mu}(P)=U_{0}^{\mu}(P)+\int_{\rho: 1} \log \rho d \mu \leqq V_{\mu}^{(0)}+\log (1+d)
$$

d'où $\stackrel{+}{V}_{0}(K)<\infty$.

Ensuite on suppose que $C_{e}^{(0)}\left(X_{1}\right)>0$ pour un ensemble $X_{1} \subset X$ relativement compact dans 2 . Soit $G \supset X_{1}$ un ensemble ouvert de diamètre $d(G)<\infty$. De la même façon que ci-dessus on a

$$
\log \underset{\dot{C}_{i}^{(0)}(G)}{1} \leqq \log \frac{1}{C_{i}^{(0)}(G)}+\log (1+d(G)),
$$

et donc

$$
\stackrel{1}{C}_{e}^{(0)}\left(X_{1}\right) \stackrel{C_{e}^{(0)}\left(X_{1}\right)}{1+d\left(X_{1}\right)},
$$

oú $d\left(X_{1}\right)$ est le diamètre de $X_{1}$. Ainsi $\stackrel{\stackrel{C}{C}}{e}^{(0)}(X) \geqslant \stackrel{+}{C}_{e}^{(0)}\left(X_{1}\right)>0$. 


\section{§2. Minoration de la capacité des ensembles produits}

2. 1. Soient $\Omega_{1}$ et $\Omega_{2}$ des espaces métriques localement compacts, et soit $\Omega=\Omega_{1} \times \Omega_{2}$ l'espace produit; si $\rho_{1}\left(P_{1}, Q_{1}\right)$ et $\rho_{2}\left(P_{2}, Q_{2}\right)$ sont les distances respectives dans $\Omega_{1}$ et $\Omega_{2}$, la distance entre $P=\left(P_{1}, P_{2}\right)$ et $Q=\left(Q_{1}, Q_{2}\right)$ dans $\Omega$ est $\operatorname{par} \rho(P, Q)=\sqrt{ } \rho_{1}^{2}\left(P_{1}, Q_{1}\right)+\rho_{2}^{2}\left(P_{2}, Q_{2}\right)$.

Lemme 1. Soient $\Omega_{1}$ et $\Omega_{2}$ des espaces métriques localement compacts. Soit $Z$ un ensemble ouvert dans l'espace produit $\Omega_{1} \times \Omega_{2}$, et soit $X$ un compact dans $\Omega_{1}$. Posons $Z_{P_{1}}=\left\{P_{2} ;\left(P_{1}, P_{2}\right) \in Z\right\}$ et supposons que $C^{(3)}\left(Z_{P_{1}}\right) \geqq b \quad(\beta \geq 0)$ pour tout $P_{1} \in X$. Alors on a

$$
\begin{gathered}
C^{(\alpha+\beta)}(Z) \geqslant \alpha^{-\alpha / 2} \beta^{-\beta / 2}(\alpha+\beta)^{(\alpha+\beta) / 2} b C^{(\alpha)}(X) \quad \text { pour } \alpha, \beta>0, \\
C^{(0)}(Z) \supseteq \sqrt{2 b C^{(0)}(X)} .
\end{gathered}
$$

On a aussi

$$
C^{(\alpha)}(Z) \geqslant C^{(\alpha)}(X)^{\pi)} \quad \text { pour } \quad \alpha \geqslant 0
$$

si $Z_{P_{1}} \neq \phi$ pour chaque $P_{1} \in X$; il ne faut pas que $C^{(3)}\left(Z_{P_{1}}\right) \geqslant b>0$.

Les mêmes relations sont vraies pour la capacité restreinte.

On peut supposer que $b>0$ et $Z$ n'est pas vide. En premier lieu nous considérons le cas où $\alpha$ et $\beta$ sont positifs. Soit $0<b^{\prime}<b$. Pour chaque point $P_{1} \in X$, on peut trouver un compact $K_{P_{1}} \subset Z_{P_{1}}$ tel que $C^{(3)}\left(K_{P_{1}}\right)>b^{\prime}$. Si on prend un voisinage ouvert $v_{P_{1}}$ de $P_{1}$ assez petit, $v_{P_{1}} \times K_{P_{1}}$ est contenu dans $Z$. Un nombre fini de tels voisinages, soient $v^{(1)}, \ldots, v^{(n)}$ couvrent $X$. Soient $K^{(1)}, \ldots, K^{(n)}$ les compacts correspondants à $v^{(1)}, \ldots, v^{(n)}$; alors $C^{(3)}\left(K^{(j)}\right)>b^{\prime}$ $(j=1, \ldots, n)$. On considère l'ensemble $\left(v^{(1)} \times K^{(1)}\right) \cup\left(\left(v^{(2)}-v^{(1)}\right) \times K^{(2)}\right)$ $\cup\left(\left(v^{(3)}-v^{(2)}-v^{(1)}\right) \times K^{(3)}\right) \cup \ldots \cup\left(\left(v^{(n)}-\bigcup_{i=1}^{n-1} v^{(i)}\right) \times K^{(n)}\right)$. Choisissons $\nu_{j} \in \widetilde{\jmath}_{K^{(j)}}$ telle que $U_{\beta}^{\nu_{j}}\left(P_{2}\right)<1 / b^{\prime}$ en tout point $P_{2}$ de $\Omega_{2}$. Désignons par $\mu_{j}$ la restriction d'une mesure quelconque $\mu \in \tilde{\mho}_{x} \grave{a} v^{(j)}-\bigcup_{i=1}^{j=1} v^{(i)}$, et posons $\sum_{j=1}^{n}\left(\mu_{j} \times \nu_{j}\right)=\lambda$. Alors $\lambda \in \mathfrak{F}_{z}$ et

$$
U_{\alpha+3}^{\hat{\alpha}}(P)=\sum_{j=1}^{n} \int_{\Omega_{2}} \int_{\Omega_{1}} \frac{d \mu_{j}\left(Q_{1}\right) d \nu_{j}\left(Q_{2}\right)}{\left\{\rho_{1}^{2}\left(P_{1}, Q_{1}\right)+\rho_{2}^{2}\left(P_{2}, Q_{2}\right)\right\}^{(\alpha+\beta) / 2}}
$$

Remarquons que, pour $s>0$ et $t>0$ quelconques,

7) (2.3) signifie (2.2) si et seulement si $C^{(0)}(X) \geq 2 b$. 


$$
\frac{s^{\alpha} t^{3}}{\left(s^{2}+t^{2}\right)^{(\alpha+\beta) / 2}}=\frac{\left(\begin{array}{c}
t \\
s
\end{array}\right)^{3}}{\left\{1+\left(\begin{array}{c}
t \\
s
\end{array}\right)^{2}\right\}^{(\alpha+\beta) / 2}} \leqq \frac{\alpha^{\alpha / 2} \beta^{\beta / 2}}{(\alpha+\beta)^{(\alpha+\beta) / 2}} ;
$$

nous désignerons la quantité à droite par $\gamma$. En posant $s=\rho_{1}\left(P_{1}, Q_{1}\right)$ et $t=\rho_{2}\left(P_{2}, Q_{2}\right)$, on aura

$$
\begin{aligned}
& \frac{1}{C^{(\alpha+\beta)}(Z)}=\sup _{P \in ! 2} U_{\alpha+\beta}^{\lambda}(P) \leq \sup _{P \in Q} \gamma \sum_{j=1}^{n} \int_{\Omega_{2}} \int_{\Omega 1} \frac{d \mu_{j}\left(Q_{1}\right)}{\rho_{1}^{\alpha}\left(P_{1}, Q_{1}\right)} \frac{d \nu_{j}\left(Q_{2}\right)}{\rho_{2}^{3}\left(P_{2}, Q_{2}\right)} \\
& \leqq r \sup _{P \in \Omega} \sum_{j=1}^{n} U_{\alpha}^{\mu_{j}}\left(P_{1}\right) U_{\beta}^{\nu}\left(P_{2}\right) \leqq \frac{r}{b^{\prime}} \sup _{P_{1} \in \Omega_{1}} U_{\alpha}^{i}\left(P_{1}\right) \text {. }
\end{aligned}
$$

Comme $\mu \in \widetilde{F_{j} x}$ peut être choisie arbitrairement, on a, en faisant $b^{\prime} \rightarrow b$,

$$
\frac{1}{C^{(\alpha+\beta)}(Z)} \leqq \frac{\gamma}{b C^{(\alpha)}(X)} .
$$

Pour établir (2.2), il suffira de remarquer que, pour $\lambda=\sum_{j=1}^{n}\left(\mu_{j} \times \nu_{j}\right)$ ci-dessus,

$$
\begin{aligned}
U_{0}^{\lambda}(P)= & \sum_{j=1}^{n} \frac{1}{2} \int_{\Omega_{2}} \int_{\Omega} \log \frac{1}{\rho_{1}^{2}\left(P_{1}, Q_{1}\right)+\rho_{2}^{2}\left(P_{2}, Q_{2}\right)} d \mu_{j}\left(Q_{1}\right) d \nu_{j}\left(Q_{2}\right) \\
= & \sum_{j=1}^{n} \frac{1}{2} \int_{\Omega_{2}} \int_{\Omega_{1}} \log \frac{\rho_{2} / \rho_{1}}{1+\left(\rho_{2} / \rho_{1}\right)^{2}} d \mu_{j} d \nu_{j}+\frac{1}{2} U_{0}^{\prime \prime}\left(P_{1}\right) \\
& +\frac{1}{2} \sum_{j=1}^{n} U_{0}^{\nu j}(P) \mu_{j}(X) \\
\leqq & \frac{1}{2} \log \frac{1}{2}+\frac{1}{2} U_{0}^{\prime}\left(P_{1}\right)+\frac{1}{2} \log \frac{1}{b^{\prime}},
\end{aligned}
$$

où $\nu_{j}$ est choisie telle que $U_{0}^{\jmath}\left(P_{2}\right) \leqq \log \frac{1}{b^{\prime}}$ dans $Q_{2}$.

Pour démontrer (2.3), on prend $\sum_{j=1}^{n}\left(v^{(j)}-\bigcup_{i=1}^{j-1} v^{(i)}\right) \times K^{(j)} \subset Z$ comme ci-dessus ; cette fois il ne faut que de $K^{(j)} \neq \phi$. Soit $\mu=\sum_{j=1}^{n} \mu_{j} \in \mathfrak{\mho}_{x}$ et $\nu_{j} \in \mathfrak{F}_{K^{(j)}}$. En posant $\lambda=\sum_{j=1}^{n}\left(\mu_{j} \times \nu_{j}\right)$, on aura

et pour $\alpha>0$

$$
U_{0}^{\lambda}(P) \leqq \int_{\Omega_{1}} \log \frac{1}{\rho_{1}\left(P_{1}, Q_{1}\right)} d \mu\left(Q_{\mathrm{i}}\right)=U_{0}^{\mu}\left(P_{1}\right)
$$

$$
U_{\alpha}^{\lambda}(P) \leqq \int_{\Omega_{1}} \frac{1}{\rho_{1}^{\alpha}\left(P_{1}, Q_{1}\right)} d \mu\left(Q_{1}\right)=U_{\alpha}^{\prime \prime}\left(P_{1}\right)
$$

On peut faire le même raisonnement pour la capacité restreinte.

Lemme 2. Soit $Z \subset \Omega_{1} \times \Omega_{2}$ un compart, et soit $X \subset \Omega_{1}$ un compact tel que, pour chaque $P_{1} \in X, C^{(3)}\left(Z_{P_{1}}\right) \geqslant b(\beta \geq 0)$. Alors 


$$
\begin{gathered}
C^{(\alpha+\beta)}(Z) \geq \alpha^{-\alpha / 2} \beta^{-3 / 2}(\alpha+\beta)^{(\alpha+\beta / 2} b C^{(\alpha)}(X) \quad \text { pour } \alpha, \beta>0, \\
C^{(0)}(Z) \geqslant \sqrt{ } 2 b C^{(0)}(X) .
\end{gathered}
$$

On a aussi

$$
C^{(\alpha)}(Z) \gtrsim C^{(\alpha)}(X)
$$

si $Z_{P_{1}} \neq \phi$ pour chaque $P_{1} \in X$; il ne faut pas que $C^{(3)}\left(Z_{P_{1}}\right) \geqslant b>0$.

Les mêmes relations sont vraies pour la capacité restreinte.

En effet, d'après la proposition 7 , on peut choisir une suite d'ensembles ouverts $\left\{G_{n}\right\}$ telle que $C^{(\alpha+\beta)}\left(G_{n}\right) \downarrow C^{(\alpha+\beta)}(Z)$. En vertu du lemme précédent on a $C^{(\alpha+\beta)}\left(G_{n}\right) \geq \gamma^{-1} b C^{(\alpha)}(X)$ si $\alpha, \beta>0$, d'où (2.4). Les autres inégalités peuvent être démontrées de la même manière.

De ce lemme on tire immédiatement le

THÉoRÈME 1. Soient $\Omega_{1}$ et $\Omega_{2}$ des espaces métriques localement compacts, $X \subset \Omega_{1}$ et $Y \subset \Omega_{2}$ des sous-ensembles quelconques, et $C_{i}^{(\alpha)}(X)$ et $C_{i}^{(\beta)}(Y)$ les capacités intérieures d'ordre $\alpha$ et d'ordre $\beta$ respectivement. Alors, pour l'ensemble produit $X \times Y$ dans l'espace métrique produit, on $a$

$$
\begin{gathered}
C_{i}^{(\alpha+\beta)}(X \times Y) \geqslant \alpha^{-\alpha / 2} \beta^{-\beta / 2}(\alpha+\beta)^{(\alpha+\beta) / 2} C_{i}^{(\alpha)}(X) C_{i}^{(\beta)}(Y) \text { pour } \alpha, \beta>0, \\
C_{i}^{(0)}(X \times Y) \geqslant 2 \sqrt{ } C_{i}^{(0)}(X) C_{i}^{(0)}(Y), \\
C_{i}^{(\alpha)}(X \times Y) \geqslant C_{i}^{(\alpha)}(X)^{s)} \quad \text { pour } \alpha \geqslant 0 \text { et pour } Y \text { non vide. }
\end{gathered}
$$

Les mêmes relations sont vraies pour la capacité intérieure restreinte.

Cependant, il reste ouvert si le fait comme le lemme 1 est vrai pour un ensemble arbitraire $Z$ et pour la capacité intérieure (restreinte).

De ce théorème on tire

Corollaire 1.

$$
\operatorname{dim}_{c i}(X \times Y) \rightleftharpoons \operatorname{dim}_{c i}(X)+\operatorname{dim}_{c i}(Y) .
$$

En vertu de la proposition 14 on obtient

Corollaire 2. Si $X$ et $Y$ sont des ensembles dans espaces euclidiens,

$$
\operatorname{dim}_{h i}(X \times Y) \gtrless \operatorname{dim}_{h i}(X)+\operatorname{dim}_{h i}(Y) .
$$

ThÉORÈMe 2. Soient $\Omega_{1}$ et $\Omega_{2}$ des espates localement compacts, soit $Z$ un 8! Voir 7). 
ensemble relativement compact dans $\Omega_{1} \times \Omega_{2}$, et soit $X \subset \Omega_{1}$. Si $Z_{P_{1}}=\left\{P_{2} ;\left(P_{1}, P_{2}\right)\right.$ $\in Z\}$ et. $C_{e}^{(\beta)}\left(Z_{P_{1}}\right) \geqq b(\beta \geqslant 0)$ pour tout $P_{1} \in X$, alors on $a$

$$
\begin{gathered}
C_{e}^{(\alpha+\beta)}(Z) \geq \alpha^{-\alpha / 2} \beta^{-\beta / 2}(\alpha+\beta)^{(\alpha+\beta) / 2} b C_{e}^{(\alpha)}(X) \quad \text { pour } \alpha, \beta>0, \\
C_{e}^{(0)}(Z) \geq \sqrt{ } 2 b C_{e}^{(0)}(X) .
\end{gathered}
$$

On a aussi

$$
C_{e}^{(\alpha)}(Z) \geqslant C_{e}^{(\alpha)}(X)^{\text {s) }} \quad \text { pour } \alpha \geqslant 0 .
$$

Les mêmes relations sont vraies pour la capacité extérieure restreinte.

En effet, soit $G \supset Z$ un ensemble ouvert, et soit $P_{1}^{*} \in X$ un point fixe quelconque. A chaque point $\left(P_{1}^{*}, P_{2}\right) \in G$, on peut trouver un ensemble produit ouvert dans $G$. Un nombre dénombrable de tels ensembles ouverts $\left\{G_{i}\right\}$ couvrent la section $\left\{\left(P_{1}^{*}, P_{2}\right) ;\left(P_{1}^{*}, P_{2}\right) \in G\right\}$ de $G$, et, pour $b^{\prime}<b$, la capacité d'ordre $\beta$ de la section $\left\{\left(P_{1}^{*}, P_{2}\right) ;\left(P_{1}^{*}, P_{2}\right) \in \bigcup_{i=1}^{n} G_{i}\right\}$ est plus grande que $b^{\prime}$ si $n$ est assez grand. Ainsi, pour chaque point, $P_{1} \in X$, il existe un ensemble produit ouvert $G_{P_{1}}$ tel que la projection $G_{P_{1}}^{\prime}$ de $G_{P_{1}}$ sur $Q_{1}$ contienne $P_{1}$ et la capacité de la projection $G_{P_{1}}^{\prime \prime}$ de $G_{P_{1}}$ sur $\Omega_{2}$ soit $>b^{\prime}$. On pose $\widetilde{G}=\bigcup_{P_{1} \in \mathrm{Y}} G_{P_{1}}$; c'est un sousensemble ouvert de $G$. Si on prend un compact $K_{1}$ quelconque dans $\bigcup_{P_{1} \in X_{1}} G_{P_{1}}^{\prime}$, on a $C^{(\alpha+\beta)}(\widetilde{G}) \geqq \gamma^{-1} b^{\prime} C^{(\alpha)}\left(K_{1}\right)$ d'après le lemme 1 . On en voit que $C^{(\alpha+\beta)}(G)$ $\geqslant C^{(\alpha+\beta)}(\widetilde{G}) \geqslant \gamma^{-1} b^{\prime} C^{(\alpha)}\left(\bigcup_{P_{1} \in \mathrm{Y}} G_{P_{1}}^{\prime}\right) \geqslant \gamma^{-1} b^{\prime} C_{e}^{(\alpha)}(X)$. Il s'en suit l'inégalité $C^{(\alpha+\beta)}(G)$ $\geqslant \gamma^{-1} b C_{e}^{(\alpha)}(X)$, d'où on obtient $C_{e}^{(\alpha+\beta)}(Z) \geqslant \gamma^{-1} b C_{e}^{(\alpha)}(X)$. Les autres relations seront démontrées de la même manière.

De ce théorème on a

Corollaire 1. Les mêmes relations que dans le théorème 1 sont vraies pour la capacité extérieure (restreinte).

Corollaire 2.

$$
\operatorname{dim}_{c e}(X \times Y) \gg \operatorname{dim}_{c e}(X)+\operatorname{dim}_{c e}(Y) .
$$

À cause de la proposition 14 on a

Corollaire 3. Si $X$ et $Y$ sont des ensembles dans espaces euclidiens,

$$
\operatorname{dim}_{h}(X \times Y) \geq \operatorname{dim}_{h}(X)+\operatorname{dim}_{h}(Y) .
$$

C'est une généralisation de (1.2) au cas où les dimensions des espaces euclidiens contenant $X$ et $Y$ respectivement sont générales. 
2. 2. Dans ce numéro nous nous occupons du cas où un espace composant est $E_{n}$, et nous considérons la mesure de Hausdorff des ensembles composants dans $E_{n}$ au lieu de la capacité. Nous voulons obtenir des résultats analogues aux théorèmes 1 et 2 , ou bien des inégalités plus précises sous forme d'intégrale prise par rapport à la mesure de Hausdorff. Cependant, nous ne pouvons démontrer que certaines relations peu satisfaisantes dans ce qui suit.

THÉoRÈme 3. Soit $X$ un ensemble dans l'espace euclidien $E_{n}, \underline{m}_{n}(X)$ sa mesure intérieure de Lebesgue et $Y$ un ensemble dans un espace métrique localement compact $\Omega$. Alors on a

$$
C_{i}^{(n+\beta)}(X \times Y) \geqslant \gamma(n, \beta) \underline{m}_{n}(X) C_{i}^{(\beta)}(Y)
$$

pour $\beta>0$ quelconque, et si $m_{n}(X)<\infty$ on a

$$
\begin{aligned}
& C_{i}^{(n)}(X \times Y) \geqslant r(n, 0) \underline{m}_{n}(X)\left[\log \frac{1}{\hat{C}_{1}^{(0)}(Y)}\right. \\
& \left.\quad+\log (1+\sqrt{2})+\frac{1}{n} \log ^{+}\left\{r(n, 0) n \underline{m}_{n}(X)\right\}\right]^{-1},
\end{aligned}
$$

où $\gamma(n, \beta)$ est une constante positive qui ne dépend que de $n$ et $\beta \geqq 0$ et $\stackrel{+}{C}_{i}^{(0)}(Y)$ est la capacite définie dans 1.7 .

Si $\underline{m}_{n}(X)=\infty$ et $C_{i}^{(0)}(Y)>0$, on a

$$
C_{i}^{(n)}(X \times Y)=\infty .
$$

Les mêmes inégalites sont valables pour les capacités intérieures restreintes $C_{i}^{(n+\beta) *}$ et $\stackrel{+}{C}_{i}^{(0) *}$.

Nous démontrerons les inégalités seulement pour $C_{i}$; le raisonnement s'applique aussi pour $C_{i}^{*}$. Un point de $E_{n}$ sera représenté par $P_{1}$ et un point de $\Omega$ par $P_{2} ; P=\left(P_{1}, P_{2}\right)$ sera dans $E_{n} \times \Omega$. On peut supposer que $Y \neq \phi$ et que $\underline{m}_{n}(X)>0$. En premier lieu considérons le cas $\beta>0$. Soit $K \subset X$ un ensemble compact de mesure $m_{n}(K)$ positive finie, et soit $\nu \in \mathfrak{F}_{Y}$. Définissons une mesure $\mu(E)$ sur les ensembles mesurables au sens de Lebesgue dans $E_{n}$ par

$$
\frac{m_{n}(E \cap K)}{m_{n}(K)}
$$

Soit $a_{n}$ l'aire de la sphère-unité dans $E_{n}$. En désignant par $a_{n}(r) r^{n-1}$ l'aire de l'intersection de $K$ avec la sphère $\sigma\left(P_{1}, r\right)$ de rayon $r$ et de centre $P_{1}$, on a . 


$$
\begin{aligned}
U_{n+3}^{+\infty}(P) & =\frac{1}{m_{n}(K)} \int_{\Omega} \int_{0}^{\infty} \frac{a_{n}(r) r^{n-1} d r d \nu\left(Q_{2}\right)}{\left\langle r^{2}+\rho^{2}\left(P_{2}, Q_{2}\right)\right\}^{(n+3) / 2}} \\
& \leqq \frac{1}{m_{n}(K)} \int_{\Omega} \frac{d \nu\left(Q_{2}\right)}{\rho^{3}\left(P_{2}, Q_{2}\right)} \int_{0}^{\infty} \frac{a_{n} t^{n-1}}{\left(1+t^{2}\right)^{(n+3) / 2}} d t .
\end{aligned}
$$

$\mathrm{Si}$ on pose la dernière intégrale égale à $K(n, \beta)$, l'inégalité s'écrit

$$
U_{n+\beta}^{\mu \times \nu}(P) \leqq \frac{K(n, \beta)}{m_{n}(K)} U_{3}^{\prime}\left(P_{2}\right) .
$$

Ainsi il en résulte (2.15).

Considérons ensuite le cas $\beta=0$. Soient $K$, $\mu$ et $\nu$ comme ci-dessus. On a

$$
\begin{aligned}
U_{n}^{\mu \times 2}(P) & =\frac{1}{m_{n}(K)} \int_{\Omega}^{\infty} d \nu \int_{0}^{\infty} \frac{a_{n}(r) r^{n-1}}{\left(r^{2}+\rho^{2}\right)^{n / 2}} d r \leqq \frac{1}{m_{n}(K)} \int_{\Omega} d \nu \int_{0}^{r_{0}} \frac{a_{n} r^{n-1}}{\left(r^{2}+\rho^{2}\right)^{n / 2}} d r \\
& =\frac{a_{n}}{m_{n}(K)} \int_{\Omega} d \nu \int_{0}^{r_{0} / \rho} \frac{t^{n-1}}{\left(1+t^{2}\right)^{n / 2}} d t,
\end{aligned}
$$

où $\boldsymbol{r}_{0}$ est le rayon de la boule dont le volume est égal à $m_{n}(K): a_{n} r_{0}^{n} / n$ $=m_{n}(K)$. Si on remarque que

$$
\frac{t^{\alpha-1}}{\left(1+t^{2}\right)^{\alpha / 2}} \leqq \frac{1}{\sqrt{1}+t^{2}} \quad \text { pour tous } \alpha \geqslant 1 \text { et } t>0
$$

on aura

d'où

$$
\begin{aligned}
U_{n}^{i, 2}(P) & \leqq \frac{a_{n}}{m_{n}(K)} \int_{\Omega} \log \left\{\frac{r_{0}}{\rho}+\sqrt{\left(\frac{r_{0}}{\rho}\right)^{2}+1}\right\} d \nu \\
& \leqq \frac{a_{n}}{m_{n}(K)}\left[\int _ { \Omega } \operatorname { l o g } \left\{\frac{1}{\rho}+\sqrt{\left.\left.\frac{1}{\rho^{2}}+1\right\} d \nu+\log { }^{+} r_{0}\right]}\right.\right. \\
& \leqq \frac{a_{n}}{m_{n}(K)}\left[\left(\int_{1 \leqq \rho}+\int_{1>\rho}\right) \log \left\{1+\sqrt{\frac{1}{\rho^{2}}+1}\right\} d \nu+\log ^{+} r_{0}\right] \\
& \leqq \frac{a_{n}}{m_{n}(K)}\left\{\int_{\Omega} \log ^{+} \frac{1}{\rho} d \nu+\log (1+\sqrt{ } 2)+\log ^{+} r_{0}\right\} \\
& \leqq \frac{a_{n}}{m_{n}(K)}\left\{U_{0}^{+}\left(P_{2}\right)+\log (1+\sqrt{2})+\frac{1}{n} \log ^{+} \frac{n m_{n}(K)}{a_{n}}\right\},
\end{aligned}
$$

$$
\left\{C_{i}^{(n)}(X \times Y)\right\}^{-1} \leqq \underset{m_{n}(K)}{a_{n}}\left\{\log \frac{1}{C_{i}^{(n)}(Y)}+\log (1+\sqrt{ } 2)+\frac{1}{n} \log \frac{n m_{n}(K)}{a_{n}}\right\} .
$$

On en conclut (2.16) si $m_{r l}(X)<\infty$. Si $\stackrel{+}{C}_{i}^{(0)}(Y)>0$ et $m_{n}(X)=\infty$, la quantité à droite tend vers 0 lorsque $m_{n}(K) \rightarrow \infty$; on $y$ tient compte de la proposition 15. Donc (2.17) est démontré.

Corollaire. Soit $X$ un ensemble dans $E_{n}$ avec $m_{n}(X)>0$ et $Y$ un ensemble dans un espace métrique localement compast $Q$ avec $C_{i}{ }^{3}(Y)>0(\beta \geq 0)$. Alors 


$$
C_{i}^{(n+3)}(X \times Y)>0 .
$$

2. 3. Puisque $C_{i}^{(n)}(X)=0$ pour tout ensemble $X$ dans $E_{n}$, le théorème 1 n'assure pas que $C_{i}^{(n+3)}(X \times Y)$ est positive, pendant que le corollaire précédent montre que $C_{i}^{(n+\beta)}(X \times Y)>0$ si $m_{n}(X)>0$ et si $C_{i}^{(3)}(Y)>0$. A ce propos nous établirons le

THÉORÈME 4. Soit $X$ un ensemble de $m_{\alpha}(X)>0 \quad(0<\alpha \leqq n)$ dans $E_{n}$, et $Y$ un ensemble de $C_{i}^{(\beta)}(Y)>0(\beta \geq 0)$ dans un espace métrique localement compact $\Omega$. Alors

$$
C_{i}^{(\alpha+\beta)}(X \times Y) \geqq \begin{cases}c(X, \alpha, \beta) C_{i}^{(\beta)}(Y) & \text { si } \beta>0, \\ \frac{1}{c(X, \alpha, 0)-c^{\prime}(X, \alpha, 0) \log C_{i}^{(0)}(Y)} & \text { si } \beta=0,\end{cases}
$$

où $c(X, \alpha, \beta)$ et $c^{\prime}(X, \alpha, \beta)$ sont des constantes positives qui ne dépendent que de $X, \alpha$ et $\beta \geqslant 0$.

On a une même relation pour $C_{i}^{*}$.

D'abord nous citons le théorème 1 de [20] dans un cas particulier.

LeMme 3. Soit « une mesure de masse totale unité à support compact dans $E_{n}$ et soit $\mu(P, r)$ la $\mu$-mesure de la boule $B(P, r)$ de centre $P$ et de rayon $r$. Pour tout entier $k>0$ nous designons par $A_{k}$ l'ensemble des points tel que chaque point $P$ satisfasse à $\mu(P, r)>k r^{\alpha}$ pour un certain nombre $r=r(P)>0$. Alors la $\mu$-valeur de $\bigcap_{k} A_{k}$ est nulle.

Nous allons démontrer le théorème. Soit $K \subset X$ un ensemble compact de $m_{\alpha}(K)$ positive finie. Nous définissons une mesure pour les ensembles boréliens dans $E_{n}$ par

$$
\mu(B)=\frac{m_{x}(K \cap B)}{m_{\alpha}(K)},
$$

et définissons $A_{k}$ comme ci-dessus; c'est un ensemble ouvert. En vertu du lemme 3 on trouve un $A_{k_{0}}$ tel que

$$
m_{\alpha}\left(A_{k_{0}}\right)<\frac{m_{\alpha}(K)}{2}
$$

L'ensemble $K_{1}=K-A_{k_{0}}$ est compact et $m_{\alpha}\left(K_{1}\right)>m_{\alpha}(K) / 2$. En chaque point $P_{1}$ de $K_{1}$ on a $\mu\left(P_{1}, r\right) \leqq k_{0} r^{\alpha}$ pour tout $r>0$. Si on pose 


$$
\mu_{1}(B)=\frac{m_{\alpha}\left(K_{1} \cap B\right)}{m_{\alpha}\left(K_{1}\right)}
$$

et désigne par $\mu_{1}\left(P_{1}, r\right)$ la $\mu_{1}$-valeur de $B\left(P_{1}, r\right)$, on aura

$$
\frac{\mu_{1}\left(P_{1}, r\right)}{r^{\alpha}} \leqq \frac{m_{\alpha}(K)}{m_{\alpha}\left(K_{1}\right)} \frac{\mu\left(P_{1}, r\right)}{r^{\alpha}} \leqq \frac{m_{\alpha}(K)}{m_{\alpha}\left(K_{1}\right)} k_{0}<2 k_{0}
$$

c'est vrai en tout point $P_{1}$ de $K_{1}$ pour tout $r>0$. Si la boule $B\left(P_{1}, r\right)$ contient un point $P_{1}^{\prime} \in K_{1}$, la boule $B\left(P_{1}^{\prime}, 2 r\right)$ contient $B\left(P_{1}, r\right)$ et on trouve

$$
\mu_{1}\left(P_{1}, r\right)<2^{\alpha+1} k_{0} r^{\alpha}
$$

en chaque point $P_{1}$ de $E_{n}$ pour tout $r>0$. En utilisant cette relation, on a, en $P=\left(P_{1}, P_{2}\right)$ dans $E_{n} \times \Omega$, pour $\nu \in \widetilde{\mho}_{Y}$ quelconque

$$
\begin{aligned}
U_{\alpha+\beta}^{\mu_{1} \times \nu}(P) & =\int_{\Omega} \int_{K_{1}} \frac{d \mu_{1} d \nu}{\left(r^{2}+\rho^{2}\right)^{(\alpha+\beta) / 2}} \leqq(\alpha+\beta) \int_{\Omega} d \nu \int_{0}^{\infty} \frac{r \mu_{1}\left(P_{1}, r\right)}{\left(r^{2}+\rho^{2}\right)^{(\alpha+\beta) / 2+1}} d r \\
& \leqq(\alpha+\beta) 2^{\alpha+1} k_{0} \int_{\Omega} \frac{d \nu}{\rho^{\beta}} \int_{0}^{\infty} \frac{t^{\alpha+1}}{\left(1+t^{2}\right)^{(\alpha+\beta) / 2+1}} d t \equiv c U_{\beta}^{\nu}\left(P_{1}\right) \quad \text { si } \quad \beta>0,
\end{aligned}
$$

où $c$ est une valeur positive qui dépend de $F, \alpha$ et $\beta$ et donc de $X, \alpha$ et $\beta$. Puisque $\nu$ est arbitraire, il en résulte le théorème dans le cas $\beta>0$.

Considérons le cas $\beta=0$ et prenons $\delta>0$. Si la distance de $P_{1}$ à $K_{1}$ est $\geqslant \delta$,

$$
U_{\alpha}^{\mu_{1} \times \nu}(P) \leqq \frac{1}{\delta^{\alpha}}
$$

en $P=\left(P_{1}, P_{2}\right)$. Si la distance est $<\delta$, en désignant par $d$ le diamètre de $K_{1}$, on a

$$
\begin{aligned}
U_{\alpha}^{\mu_{1} \times \nu}(P) & =\int_{\Omega}\left\{(\delta+d)^{2}+\rho^{2}\right\}^{\alpha / 2}+\alpha \int_{\Omega} d \nu \int_{0}^{\delta+d} \frac{r \mu_{1}\left(P_{1}, r\right)}{\left(r^{2}+\rho^{2}\right)^{(\alpha / 2)+1}} d r \\
& \leqq \frac{1}{(\delta+d)^{\alpha}}+\alpha 2^{\alpha+1} k_{0} \int_{\Omega} d \nu \int_{0}^{\delta+d} \frac{r^{1+\alpha}}{\left(r^{2}+\rho^{2}\right)^{(\alpha / 2)+1}} d r \\
& =\frac{1}{(\delta+d)^{\alpha}}+\alpha 2^{\alpha+1} k_{0} \int_{\Omega} d \nu \int_{0}^{(\delta+d) / /} \frac{t^{1+\alpha}}{\left(1+t^{2}\right)^{(\alpha / 2)+1}} d t \\
& \leqq \frac{1}{(\delta+d)^{\alpha}}+\alpha 2^{\alpha+1} k_{0} \int_{0} d \nu \int_{0}^{(\delta+d) / \rho} \frac{d t}{\sqrt{1+t^{2}}} \\
& \leqq \frac{1}{(\delta+d)^{\alpha}}+\alpha 2^{\alpha+1} k_{0}\left\{U_{0}^{\nu}\left(P_{2}\right)+\log (1+v 2)+\log ^{-}(\delta+d)\right\} .
\end{aligned}
$$

Donc en tout point $P$ de $E_{n} \times \Omega$

$$
U_{\alpha}^{\mu_{1} \times \nu}(P) \leqq \frac{1}{\delta^{\alpha}}+\alpha 2^{\alpha+1} k_{0}\left\{\log \frac{1}{C_{i}^{(0)}(Y)}+\log (1+v 2)+\log ^{+}(\delta+d)\right\} .
$$


On peut choisir $\delta>0$ arbitrairement; par exemple, si on pose $\delta=d$,

$$
C_{i}^{(\alpha)}(X \times Y) \geq \frac{1}{\delta^{-\alpha}+\alpha 2^{\alpha+1} k_{0}\left\{\log (1+\sqrt{2})+\log ^{+}(2 d)\right\}-\alpha 2^{\alpha+1} k_{0} \log \dot{C}_{i}^{(\alpha)}(Y)} .
$$

Ainsi le théorème est démontré.

On peut obtenir une même relation pour $C_{i}^{*}$ de la même façon.

Enfin on pose

Question. Supposons que $C_{i}^{\left(\alpha^{\prime}\right)}(X)>0$ pour tout $\alpha^{\prime}<\alpha$ et que $C_{i}^{(\beta)}(Y)>0$. Alors, $C_{i}^{(\alpha+\beta)}(X \times Y)$ est-elle toujours positive?

C'est le cas par le théorème 4 pour un ensemble $X \subset E_{n}$ de $\underline{m}_{\alpha}(X)>0$ même si $C_{i}^{(\alpha)}(X)=0$.

\section{§. Majoration de la capacité}

3. 1. La proposition 13 et l'égalité de (1.3) montrent qu'il arrive que, pour certains ensembles fermés $X, Y \subset E_{1}$,

$$
\operatorname{dim}_{c}(X)+\operatorname{dim}_{c}(Y)<\operatorname{dim}_{c}(X \times Y) .
$$

Dans le paragraphe 4 on trouvera d'autres tels exemples qui montrent qu'il n'existe aucune limitation supérieure finie pour la dimension capacitaire des ensembles produits qui ne dépend que des dimensions capacitaires des ensembles composants. Donc nous ne pouvons pas trouver une majoration de la capacité des ensembles produits au moyen de la capacité ou de la mesure de Haussorff, sauf dans ertains cas particuliers dont nous allons nous occuper dans ce paragraphe.

THÉORÈmE 5. Soit $X \subset E_{n}(n \geq 1)$ un ensemble borélien de $m_{a}(X)<\infty$ tel que la densité inférieure

$$
d\left(P_{1}\right)=\lim _{r \rightarrow 0} \frac{m_{\alpha}\left(P_{1}, r\right)}{r^{\alpha}}
$$

soit positive en tout point $P_{1} \in X$, où $m_{\alpha}\left(P_{1}, r\right)$ signifie la $m_{\alpha}$-mesure de la partie de $X$ dans la boule $B\left(P_{1}, r\right)$. Soit $Y$ un ensemble $\beta$-polaire $(\beta \geqslant 0)$ dans un espace métrique localement compact $\Omega$. Alors $X \times Y$ est un ensemble $(\alpha+\beta)$. polaire dans $E_{n} \times \Omega$.

En effet, on peut supposer que $X$ et $Y$ sont relativement compacts dans $E_{n}$ et $\Omega$ respectivement. Soit $\nu \in \mathcal{F}_{\Omega}$ une mesure telle que $U_{\beta}^{\nu}\left(P_{2}\right)=\infty$ partout sur $Y$, et soit $P_{1}$ un point quelconque de $X$. Prenons $r_{1}>0$ tel que 


$$
\frac{m_{\alpha}^{\prime}\left(P_{1}, r\right)}{r^{\alpha}}>\frac{d\left(P_{1}\right)}{2}
$$

pour tout $r \in\left(0, r_{1}\right)$, et posons $\mu(B)=m_{\alpha}(B \cap X)$.

Lorsque la $\nu$-mesure de $P_{2}$ est nulle, on a, en $P=\left(P_{1}, P_{2}\right) \in X \times Y$,

$$
\begin{aligned}
U_{\alpha+\beta}^{\mu \times \nu}(P) & =\int_{\Omega} d \nu \int_{x} \frac{d m_{\alpha}\left(P_{1}, r\right)}{\left(r^{2}+\rho^{2}\right)^{(\alpha+\beta) / 2}}=(\alpha+\beta) \int_{\Omega} d \nu \int_{0}^{\infty} \frac{m_{\alpha}\left(P_{1}, r\right) r}{\left(r^{2}+\rho^{2}\right)^{(\alpha+\beta) / 2+1}} d r \\
& \geqslant \frac{(\alpha+\beta) d\left(P_{1}\right)}{2} \int_{\Omega} d \nu \int_{0}^{r_{1}} \frac{r^{\alpha+1}}{\left(r^{2}+\rho^{2}\right)^{(\alpha+\beta) / 2+1}} d r \\
& \geqslant \frac{(\alpha+\beta) d\left(P_{1}\right)}{2} U_{\beta}^{\nu}\left(P_{2}\right) \int_{0}^{r_{1} / d} \frac{t^{\alpha+1}}{\left(1+t^{2}\right)^{(\alpha+\beta) / 2+1}} d t
\end{aligned}
$$

si $\beta>0$, où $d$ est le diamètre du support $S_{\nu}$. Si $\beta=0$, on a

$$
\begin{aligned}
U_{\alpha}^{\mu \times \nu}(P) & \geqq \frac{\alpha}{2} d\left(P_{1}\right) \int_{\Omega} d \nu \int_{0}^{r_{1} / p} \frac{t^{\alpha+1}}{\left(1+t^{2}\right)^{\alpha / 2+1} d t} \\
& \geqq \frac{\alpha}{2} d\left(P_{1}\right) \int_{\rho \leqq r_{1}} d \nu \int_{0}^{r_{1} / \rho} \frac{t^{\alpha+1}}{\left(1+t^{2}\right)^{\alpha / 2+1}} d t \\
& \geqq \frac{\alpha d\left(P_{1}\right)}{2^{2+\alpha / 2}} \int_{\rho \leqq r_{1}} d \nu \int_{1}^{r_{1} / \rho} d t \geq \frac{\alpha d\left(P_{1}\right)}{2^{2+\alpha / 2}} \int_{\Omega} \log \frac{r_{1}}{\rho} d \nu \\
& =\frac{\alpha d\left(P_{1}\right)}{2^{2+\alpha / 2}}\left\{U_{0}^{\prime}\left(P_{2}\right)+\log r_{1}\right\} .
\end{aligned}
$$

Comme $\quad U_{\beta}^{\nu}\left(P_{2}\right)=\infty, \quad U_{\alpha+\beta}^{\mu \times \nu}(P)=\infty \quad(\beta \geqslant 0) \quad$ en $\quad P=\left(P_{1}, P_{2}\right) \in X \times Y$ si $\nu\left(\left\{P_{2}\right\}\right)=0$.

Supposons ensuite que la $\nu$-mesure de $P_{2} \in Y$ soit positive : $\nu_{\nu}\left(\left\{P_{2}\right\}\right)=\nu_{0}>0$. Alors en $P_{1} \times P_{2} \in X \times Y$ on a, pour $\varepsilon>0$ quelconque,

$$
\begin{aligned}
U_{\alpha+\beta}^{\mu \times \nu}(P) & \geqslant \nu_{0} \int_{X} \frac{d m_{\alpha}\left(P_{1}, r\right)}{r^{\alpha+\beta}} \geqslant \nu_{0} \int_{X} \frac{d m_{\alpha}\left(P_{1}, r\right)}{(r+\varepsilon)^{\alpha+\beta}} \\
& =(\alpha+\beta) \nu_{0} \int_{0}^{\infty} \frac{m_{\alpha}\left(P_{1}, r\right)}{(r+\varepsilon)^{\alpha+\beta+1}} d r \\
& \geqslant \frac{(\alpha+\beta) \nu_{0} d\left(P_{1}\right)}{2} \int_{0}^{r_{1}} \frac{r^{\alpha}}{(r+\varepsilon)^{\alpha+\beta+1}} d r \\
& =\frac{(\alpha+\beta) \nu_{0} d\left(P_{1}\right)}{2 \varepsilon^{3}} \int_{0}^{r_{1} / \xi} \frac{t^{\alpha}}{(1+t)^{\alpha+\beta+1}} d t \rightarrow \infty
\end{aligned}
$$

lorsque $\varepsilon \rightarrow 0$ si $\beta>0$. Si $\beta=0$ on a encore

$$
U_{\alpha}^{\mu+\nu}(P) \triangleq \alpha \nu_{0} \int_{0}^{r_{1}} \frac{r^{\alpha}}{(r+\varepsilon)^{\alpha+1}} d r=\alpha \nu_{0} \int_{0}^{r_{1} / \varepsilon} \frac{t^{\alpha}}{(1+t)^{\alpha+1}} d t \rightarrow \infty
$$

lorsque $\varepsilon \rightarrow 0$, car 


$$
\int_{0}^{\infty} \frac{t^{\alpha}}{(1+t)^{\alpha+1}} d t=\infty
$$

Ainsi $U_{a+3}^{\mu \times b}(P)=\infty$ en tout point de $X \times Y$ et donc $X \times Y$ est un ensemble $(\alpha+\beta)$-polaire.

3. 2. Dans ce numéro on considère le cas particulier où $X$ est un cube non-dégénéré dans $E_{n}$. Nous trouverons une majorante de $C_{i}^{(n+\beta) *}(X \times Y)$ au moyen de $C_{i}^{(\beta) *}(Y)$ et de la longueur des côtés de $X$.

On commence avec le

Lemme 4. Soit $X$ un cube fermé de côté $l$ dans $E_{n}(n \geqslant 1)$, et soit $\mu$ la mesure definie par $\mu(B)=m_{n}(B \cap X) / m_{n}(X)$ pour les ensembles boréliens $B$. Soit $\Omega$ un espace métrique localement compact et soit $\nu$ une répartition de masseunité dans $\Omega$. Alors, en $P=\left(P_{1}, P_{2}\right) \in X \times S_{\nu}$, on a

$$
U_{\alpha+\beta}^{\mu \times \nu}(P) \geqslant \begin{cases}c_{n}\left\{U_{\beta}^{\nu}\left(P_{2}\right)-1\right\}^{+} /\left(1+l^{2}\right)^{(n+\beta) / 2} & \text { si } \beta>0, \\ n c_{n} \stackrel{+}{U}_{0}^{\nu}\left(P_{2}\right) /\left(1+l^{2}\right)^{n / 2} & \text { si } \beta=0,\end{cases}
$$

où $c_{n}$ est une constante positive dependant seulement de $n$.

Il est visible qu'il existe une constante $c_{n}$ qui dépend seuelement de $n$ telle qu'en tout point $P_{1}$ de $X$ on ait

$$
\mu\left(P_{1}, r\right) \gtrsim c_{n} r^{n} / l^{n}
$$

pour tout $r \in[0, l]$.

En premier lieu supposons que la $\nu$-mesure de $P_{2}$ soit nulle. On aura en $P \in X \times S_{\downarrow}$

$$
\begin{aligned}
U_{n+\beta}^{\mu \times \nu}(P) & \geqslant \int_{\Omega} d \nu \int_{0}^{l} \frac{d \mu\left(P_{1}, r\right)}{\left(r^{2}+\rho^{2}\right)^{(n+\beta) / 2}} \\
& =\int_{\Omega} d \nu\left\{\frac{\mu\left(P_{1}, l\right)}{\left(l^{2}+\rho^{2}\right)^{(n+\beta) / 2}}+(n+\beta) \int_{0}^{l} \frac{\mu\left(P_{1}, r\right) r}{\left(r^{2}+\rho^{2}\right)^{(n+\beta) / 2+1}} d r\right\} \\
& \geqq \int_{\Omega} d \nu\left\{\frac{c_{n}}{\left(l^{2}+\rho^{2}\right)^{(n+\beta) / 2}}+\frac{(n+\beta)}{l^{n}} \int_{0}^{l} \frac{c_{n} r^{n+1}}{\left(r^{2}+\rho^{2}\right)^{(n+\beta) / 2+1}} d r\right\} \\
& =\frac{c_{n}}{l^{n}} \int_{\Omega} d \nu \int_{0}^{l} \frac{n r^{n-1}}{\left(r^{2}+\rho^{2}\right)^{(n+\beta) / 2}} d r=n c_{n} \int_{\Omega} \frac{d \nu}{\rho^{\beta}} \int_{0}^{1 / \rho} \frac{t^{n-1}}{\left(1+l^{2} t^{2}\right)^{(n+\beta) / 2}} d t \\
& \geqslant \frac{c_{n}}{\left(1+l^{2}\right)^{(n+\beta) / 2}} \int_{\rho \equiv 1} \frac{d \nu}{\rho^{\beta}} \geq \frac{c_{n}}{\left(1+l^{2}\right)^{(n+\beta) / 2}}\left\{U_{\beta}^{\nu}\left(P_{2}\right)-1\right\}^{+} \quad \text { si } \beta>0,
\end{aligned}
$$

et si $\beta=0$ on aura 


$$
U_{n}^{\mu \times \nu}(P) \supseteq \frac{n c_{n}}{\left(1+l^{2}\right)^{n / 2}} \int_{\rho \leqq 1} d \nu \int_{1}^{1 / \rho} \frac{d t}{t}=\frac{n c_{n}}{\left(1+l^{2}\right)^{n / 2}} \stackrel{+}{U}_{0}^{\nu}\left(P_{2}\right)
$$

Dans le cas où la $\nu$-mesure de $P_{2}$ est positive, on peut montrer que $U_{n+\beta}^{\mu \times \nu}(P)$ $=\infty$ de la même manière que dans la démonstration du théorème précédent. Ainsi le lemme est démontré.

Remarque. Si le diamètre $d$ de $Y$ est fini, on peut montrer pour toute $\nu$ portée par $Y$ que sur $X \times S_{\nu}$

$$
U_{n+\beta}^{\mu \times \nu}(P) \geqq k(n, \beta, l, d) U_{3}^{\nu}\left(P_{2}\right) \quad \text { pour } \beta>0,
$$

où $k(n, \beta, l, d)$ est une constante positive qui dépend de $n, \beta, l$ et $d$.

THÉORÈme 6. Soit $X$ un cube fermé de côté $l$ dans $E_{n}(n \geqslant 1)$ et soit $Y$ un ensemble dans un espace métrique localement compact $\Omega$. Alors, avec la même constante $c_{n}$ que dans le lemme précédent, on a

$$
C_{i}^{(n+\beta) *}(X \times Y) \leqq \frac{\left(1+l^{2}\right)^{(n+\beta) / 2}}{c_{n}\left\{\frac{1}{C_{i}^{(\beta) *}(Y)}-1\right\}^{+}} \text {si } \beta>0
$$

et

$$
C_{i}^{(n) *}(X \times Y) \leqq \frac{\left(1+l^{2}\right)^{n / 2}}{n c_{n} \log \frac{1}{C_{i}^{(0) *}(Y)}} \quad \text { si } \beta=0
$$

On a les mêmes inégalités pour $C_{e}^{*}$.

En premier lieu supposons que $C_{i}^{(\beta) *}(Y)>0$. Soit $K$ un sous-compact de $Y$ tel que $C_{i}^{(\beta) *}(K)>0$. Soit $\varepsilon>0$. Dans le cas $\beta>0$, il existe, d'après la proposition 11, une répartition $\nu_{0}$ de masse-unité telle que

$$
U_{\beta}^{\nu_{0}}\left(P_{2}\right) \geqslant \frac{V_{\beta}^{*}(K)}{1+\varepsilon} \quad \text { sur } K .
$$

Notons $\mu(B)=m_{n}(B \cap X) / l^{n}$. Alors par le lemme précédent on a

$$
U_{n+\beta}^{\mu \times \nu_{0}}(P) \geqq c_{n}\left\{U_{\beta}^{\nu_{0}}\left(P_{2}\right)-1\right\}^{r} /\left(1+l^{2}\right)^{(n+\beta) / 2} .
$$

Nous intégrons des deux côtés par rapport à une mesure $\nu \in \widetilde{F} x \times y$, Le théorèrne de Fubini donne lieu à

$$
\begin{aligned}
\int_{\Omega} U_{n+\beta}^{\prime} d\left(\mu \times \nu_{0}\right) & =\int_{\Omega} U_{n+\beta}^{\mu \times \nu_{0}} d \nu \geq c_{n}\left\{\int_{\Omega} U_{\beta}^{\nu_{1}} d \nu-1\right\}^{+} /\left(1+l^{2}\right)^{(n+\beta) / 2} \\
& \geq c_{n}\left\{\frac{V_{\beta}^{*}(K)}{1+\xi}-1\right\}^{+} /\left(1+l^{2}\right)^{(n+\beta) / 2}
\end{aligned}
$$


Il en résulte que

$$
C_{i}^{(n+\beta) *}(X \times K) \leqq \frac{\left(1+l^{2}\right)^{(n+\beta) / 2}}{c_{n}\left\{\frac{1}{C_{i}^{(\beta) *}(Y)}-1\right\}^{+}} .
$$

Puisqu'un ensemble compact dans $X \times Y$ est contenu dans le produit de $X$ et un certain ensemble compact dans $Y$, nous avons enfin

$$
C_{i}^{(n+\beta) *}(X \times Y) \leqq \frac{\left(1+l^{2}\right)^{(n+\beta) / 2}}{c_{n}\left\{\frac{1}{C_{i}^{(\beta) *}(Y)}-1\right\}^{+}} .
$$

Considérons le cas où $C_{i}^{(\beta) *}(Y)=0$. Alors tout sous-ensemble compact $K$ de est $\beta$ polaire et, d'après le théorème $\bar{j}, X \times K$ est $(n+\beta)$-polaire, d'où $C_{i}^{(n+* *}(X \times K)=0$ et donc $C_{i}^{(n+\beta) *}(X \times Y)=0$.

Dans le cas $\beta=0$, on fait un raisonnement analogue en utilisant la proposition $1^{\prime}$ dans 1.7 et on obtient (3.2).

Pour démontrer les inégalités pour la capacité extérieure, considérons un evsemble $Y_{1} \subset Y$ qui est relativement compact dans $\Omega$. Étant donné $\varepsilon, 0<\varepsilon<1$, i) existe un ensemble ouvert $G \supset Y_{1}^{\prime}$ tel que $C_{i}^{(\beta) *}(G)<C_{e}^{(\beta) *}\left(Y_{1}\right)+\varepsilon$ si $\beta>0$ et $\stackrel{+}{C}_{i}^{(0) *}(G)<\stackrel{C}{C}_{i}^{(0) *}\left(Y_{1}\right)+\varepsilon$ si $\beta=0$. Prenons un cube $X_{1}$ de côté $l+\varepsilon$ contenant $X$ dans son intérieur. Alors $X_{1} \times G$ contient $X \times Y_{1}$, et on a

$$
\begin{aligned}
C_{e}^{(n+\beta) *}\left(X \times Y_{1}\right) & \leqq C_{i}^{(n+\beta) *}\left(X_{1} \times G\right) \\
& \leqq \frac{\left\{1+(l+\varepsilon)^{2}\right\}^{(n+\beta) / 2}}{c_{n}\left\{\frac{1}{C_{i}^{(\beta) *}\left(Y_{1}\right)+\varepsilon}-1\right\}^{+}}<\frac{\left\{1+(l+\varepsilon)^{2}\right\}^{(n+\beta) / 2}}{c_{n}\left\{\frac{1}{C_{e}^{(\beta) *}(Y)+\varepsilon}-1\right\}^{+}}
\end{aligned}
$$

si $\beta>0$ et

$$
C_{e}^{(n) *}\left(X \times Y_{1}\right) \leqq C_{i}^{(n) *}\left(X_{1} \times G\right) \leqq \frac{\left\{1+(l+\varepsilon)^{2}\right\}^{n / 2}}{n c_{n} \log \frac{1}{C_{i}^{(0) *}(Y)+\varepsilon}}
$$

si $\beta=0$. Comme un sous-ensemble de $X \times Y$ qui est relativement compact dans $E_{n} \times \Omega$ est contenu dans un certain ensemble produit de forme $X \times Y_{1}$, on peut facilement en déduire les inégalités cherchées.

Remarque. Dans (3.1) et (3.2) les quantités à droite ne tendent pas vers 0 lorsque $l \rightarrow 0$. En effet, si on prend pour $X$ un segment de longueur $l$, et pour $Y$ un carré dans un plan, alors pour $\beta, 0 \leqq \beta_{\text {s }}<1$,

$$
0<C_{i}^{(1+\beta) *}(Y) \leqq C_{i}^{(1+\beta) *}(X \times Y) \text {. }
$$


3. 3. On démontrera une généralisation du théorème de Deny-Lelong [10] cité dans l'introduction.

THÉORÈME 7. soit $X$ un cube fermé dans $E_{n}(n \geqslant 1)$ et soit $Y$ un ensemble dans un espace métrique localement compact 2 . Alors $C_{i}^{(n+\beta)}(X \times Y)>0$ si et seulement si $C_{i}^{(3)}(Y)>0(\beta \geqq 0)$. Il en est même de $C_{e}$.

À cause de la proposition 5 , il revient au même de considérer $C$ et de considérer $C^{*}$. Le théorème 3 et la proposition 15 montrent que si $C_{i}^{\prime 3 \prime}(Y)>0$ $(\beta \geqslant 0)$ alors $C_{i}^{(n+3)}(X \times Y)>0$, et le théorème 6 et la proposition 15 assurent que si $C_{i}^{(\beta) *}(Y)=0\left(C_{e}^{(\beta) *}(Y)=0\right.$ resp. $)$ alors $C_{i}^{(n+\beta) *}(X \times Y)=0\left(C_{e}^{(n+\beta) *}(X \times Y)=0\right.$ resp.). Il reste seulement à démontrer $C_{e}^{(n+\beta)}(X \times Y)>0$ de l'hypothèse $C_{e}^{(\text {) }}(Y)>0(\beta \geqslant 0)$.

Donc nous supposons que $C_{e}^{(\beta) *}(Y)>0(\beta \geqslant 0)$. Alors on peut trouver un ensemble $Y_{1} \subset Y$ relativement compact dans $\Omega$ tel que $C_{e}^{(3) *}\left(Y_{1}\right)>0$; si $\beta=0$, d'après les propositions $\bar{j}$ et $1 \bar{j} \dot{C}_{e}^{(0) *}\left(Y_{1}\right)>0$. Soit $G$ un ensemble ouvert quelconque contenant $Y_{1}$ et soit $X_{0}$ l'intérieur de $X$. L'ensemble $X_{0} \times G$ est ouvert et d'après le théorème 3

$$
C_{i}^{(n+\beta) *}\left(X_{0} \times G\right) \geqq \gamma(n, \beta) l^{n} C_{i}^{(3) *}(G) \geqq \gamma(n, \beta) l^{n} C_{e}^{(\beta) *}\left(Y_{1}\right)>0 \quad \text { si } \beta>0
$$

et

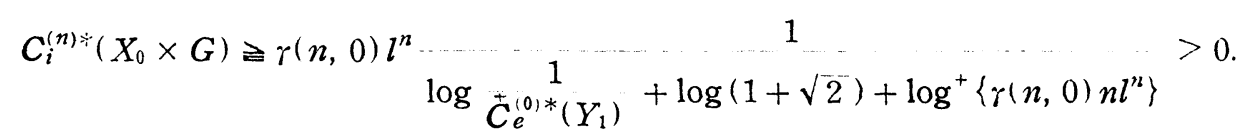

Cela étant, soit $V$ un ensemble ouvert quelconque contenant $X \times Y_{1}$, et soit $V_{P_{1}}$ la section de $V$ de l'abscisse $P_{1}: V_{P_{1}}=\left\{P_{2} ;\left(P_{1}, P_{2}\right) \in V\right\}$. Puisque $X$ est compact, il est facile de voir que $G_{1}=\bigcap_{P_{1} \in X} V_{P_{1}}$ est un ensemble ouvert dans $\Omega$. Evidemment $G_{1}$ contient $Y$ et les inégalités ci-dessus sont vraies pour $X_{0} \times G_{1}$. Ainsi $C_{i}^{(n+\beta) *}(V)$ qui est $\geqslant C_{i}^{(n+\beta) *}\left(X_{0} \times G_{1}\right)$ possède une minorante positive finie qui ne dépend que de $C_{e}^{(\beta) *(}\left(Y_{1}\right)>0, n$ et $l$. Puisque $C_{e}^{(n-\beta) *}(X \times Y)$ $\geqslant C_{e}^{(n+\beta) *}\left(X \times Y_{1}\right)=\inf _{1} C_{i}^{(n+\beta) *}(V)$, on conclut que $C_{e}^{(n+\beta) *}(X \times Y)>0$.

\section{§. Dimension des ensembles produits}

4. 1. Soit $X$ un compact dans un espace euclidien $E_{n}$ et soit $Y$ un compact dans un autre espace euclidien $E_{m}$. Le corollaire $2 \mathrm{du}$ théorème 1 énonce que

$$
\operatorname{dim}(X)+\operatorname{dim}(Y) \leqq \operatorname{dim}(X \times Y) .
$$


Si on renferme $X$ par un cube dans $E_{n}$, il résulte du théorème 6 que $\operatorname{dim}(X \times Y) \leqq n+\operatorname{dim}(Y)$. Pour la même raison on a $\operatorname{dim}(X \times Y) \leqq m$ $+\operatorname{dim}(X)$, et donc

$$
\operatorname{dim}(X \times Y) \leqq \min \{n+\operatorname{dim}(Y), m+\operatorname{dim}(X)\} .
$$

Il est évident qu'il existe certains cas qui donnent lieu à l'égalité dans (4.1). Dans ce paragraphe on construira un exemple qui réalise l'égalité dans (4.2).

THÉORÈME 8. Etant donnés des entiers $n$ et $m$ et des nombres $\alpha, 0<\alpha \leqq n$, et $\beta, 0<\beta \leqq m$, il existe un compact $X \subset E_{n}$ de dimension $\alpha$ et un compact $Y \subset E_{m}$ de dimension $\beta$ tels que

$$
\operatorname{dim}(X \times Y)=\min (n+\beta, m+\alpha) .
$$

Nous démontrerons ce théorème dans 4.3-4.5.

4. 2. Dans ce numéro nous répétons la définition, donnée dans [24], des ensembles de Cantor généralisés dans $E_{n}(n \geqslant 1)$. D'abord nous nous occupons de $E_{1}$.

Soient $k_{1}, k_{2}, \ldots$ des nombres entiers supérieurs à 1 et soient $p_{1}, p_{2}, \ldots$ des nombres finis quelconques également supérieurs à 1 . On pose $l_{q}=1 /\left(k_{q} p_{q}\right)$. Soit $I$ un intervalle de longueur $d>0$. On enlève de $I\left(k_{q}-1\right)$ intervalles de même longueur tels qu'il reste $k_{q}$ intervalles de même longueur $l_{q} d$. On appelle cette opération la $q$-opération appliquée à $I$. On commence par appliquer l'lopération à $[0,1]$, on applique la 2 -opération à chacun des intervalles $I_{1 \mathrm{v}}$ $\left(1 \leqq \nu \leqq k_{1}\right)$ qui restent, puis on applique la 3-opération à chacun des intervalles $I_{2 \lambda}\left(1 \leqq \lambda \leqq k_{1} k_{2}\right)$ qui restent, et ainsi de suite. On appellera l'ensemble limite restant un ensemble de Cantor généralisé dans $E_{1}$, et le notera $F=F\left(k_{q}, p_{q}\right)$.

Dans $E_{n}=\left\{\left(x_{1}, \ldots, x_{n}\right)\right\}$, soit $F_{j}=F\left(k_{q}^{(j)}, p_{q}^{(j)}\right)$ un ensemble de Cantor généralisé défini sur l'axe des $x_{j}$. Nous appellerons l'ensemble produit $F=F_{1} \times \ldots \times F_{n}$ un ensemble de Cantor généralisé dans $E_{n}(n \geqslant 2)$. Il sera appelé symétrique si $F_{1}=\ldots=F_{n}$.

Dans [24] l'auteur a démontré le théorème suivant:

Soit $F=F\left(k_{q}, p_{q}\right) \times \ldots \times F\left(k_{q}, p_{q}\right)$ un ensemble de Cantor généralisé symétrique dans $E_{n}(n \geqslant 1)$. Pour qu'il soit d' $\alpha$-capacité nulle $(0 \leqq \alpha<n)$, il faut et il suffit que 


$$
\sum_{q=1}^{n} \frac{\left(p_{1} \cdots p_{q}\right)^{\alpha}}{\left(k_{1} \cdots k_{q}\right)^{n-\alpha}}=\infty \quad(0<\alpha<n)
$$

ou

$$
\sum_{q=1}^{\infty} \frac{\log p_{q}}{\left(k_{1} \cdots k_{q}\right)^{n}}=\infty \quad(\alpha=0) .
$$

4. 3. Démonstration du théorème 8. En premier lieu considérons le cas $\alpha<n$ et $\beta<m$. Prenons dans $E_{n}$ un ensemble de Cantor généralisé symétrique $F=F\left(k_{q}, p_{q}\right) \times \ldots \times F\left(k_{q}, p_{q}\right)$ de dimension $\alpha$, et prenons dans $E_{m} F^{\prime}=F\left(k_{q}^{\prime}, p_{q}^{\prime}\right)$ $\times \ldots \times F\left(k_{q}^{\prime}, p_{q}^{\prime}\right)$ de dimension $\beta ; k_{q}, p_{q}, k_{q}^{\prime}$ et $p_{q}^{\prime}$ seront déterminés ultérieurement. Les quantités pour $F^{\prime}$ correspondant à des quantités pour $F$ seront notées avec l'accent '. On peut supposer, sans limiter la généralité, que $n+\beta \geqslant m+\alpha$. Nous allons évaluer la capacité d'ordre $m+\alpha^{\prime}, 0<\alpha^{\prime}<\alpha$, de l'ensemble produit $F \times F^{\prime}$.

Nous désignons les coordonnées dans $E_{n}$ par $x_{1}, \ldots, x_{n}$ et dans $E_{m}$ par $x_{1}^{\prime}, \ldots, x_{m}^{\prime}$. Désignons par $\left\{I_{q}^{(j)}\right\}$ les intervalles qui restent lorsque la $q$ opération est appliquée sur l'axe des $x_{j}$, par $\lambda_{q}$ leur longueur et par $\delta_{q}$ la longueur des intervalles enlevés lors de la $q$-opération;

$$
\lambda_{q}=\frac{1}{k_{1} \cdots k_{q} p_{1}} \cdots \bar{p}_{q}^{-}, \quad \delta_{q}=\lambda_{q-1} \frac{1-p_{q}^{-1}}{k_{q}-1}=\frac{p_{q}-1}{k_{1} \cdots k_{q-1}\left(k_{q}-1\right) p_{1} \cdots p_{q}}
$$

Nous avons les quantités correspondantes et les relations analogues sur l'axe des $x_{j^{\prime}}^{\prime}$.

Soit $\mu$ une répartition de masse-unité sur $F \times F^{\prime}$ et soit $U_{m+\alpha^{\prime}}^{\mu}(P)$ le potentiel engendré par $\mu$ dans $F \times F^{\prime}$. Nous posons

$$
\mu\left(I_{q \nu_{1}}^{(1)} \times \ldots \times I_{q \nu_{n}}^{(n)} \times I_{q \nu_{1}}^{(1)} \times \ldots \times I_{q \nu_{m}^{\prime}}^{(m)} \times \mu_{q}^{\prime}\left(\nu_{1}, \ldots, \nu_{n}, \nu_{1}^{\prime}, \ldots, \nu_{m}^{\prime}\right) .\right.
$$

Considérons les points $\left\{P_{\kappa}^{(q)}\right\}, \kappa=1, \ldots, k_{1}^{n} \cdots k_{q-1}^{n}\left(k_{q}-1\right)^{n} k_{1}^{\prime \prime \prime} \cdots k_{q-1}^{\prime m}\left(k_{q}^{\prime}-1\right)^{m}$ $\equiv M_{q}$, tels que la projection de chaque point sur tout axe coïncide avec un certain point placé au centre d'un intervalle qu'on enlève lors de la $q$-opération. On désigne $\operatorname{par}\left(P_{\kappa}^{(q)}, I_{q \nu_{1}}^{(1)} \times \ldots \times I_{q \nu_{n}}^{(n)} \times I_{q \nu^{\prime}}^{(1)} \times \ldots \times I_{q l^{\prime} m^{\prime}}^{(m)}\right)$ la distance de $P_{\kappa}^{(q)}$ à $I_{q \nu_{1}}^{(1)} \times \ldots \times I_{q \nu_{n}}^{(n)} \times I_{q \nu_{1}^{\prime}}^{(1)} \times \ldots \times I_{q \nu^{\prime}{ }^{\prime}}^{(m)} . \quad \mathrm{Si}$

$$
\Delta_{\kappa}^{(q)}\left(\nu_{1}, \ldots, \nu_{n}, \nu_{1}^{\prime}, \ldots, \nu_{m}^{\prime}\right) \leqq \rho\left(P_{\kappa}^{(q)}, I_{q \nu_{1}}^{(1)} \times \ldots \times I_{q \nu_{n}}^{(n)} \times I_{q \nu_{1}}^{(1)} \times \ldots \times I_{q \nu_{m}^{\prime}}^{(m)} \times,\right.
$$

on a

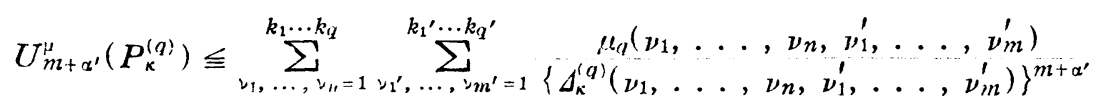

Posons $u_{q}$ égal à la moyenne des valeurs $\left\{U_{m+\alpha^{\prime}}\left(P_{\kappa}^{(q)}\right)\right\}, \kappa=1, \ldots, M_{q}$. Alors 


$$
\begin{aligned}
u_{q} & \leqq \frac{1}{M_{q}} \sum_{\nu_{1}, \ldots, \nu_{n}=1}^{k_{1} \ldots k_{q}} \sum_{\alpha_{1}}^{k_{1}^{\prime} \ldots k_{q^{\prime}}} \mu_{\nu_{n^{\prime}}=1}^{M_{q}}\left(\nu_{1}, \ldots, \nu_{n}, \nu_{1}^{\prime}, \ldots, \nu_{m}^{\prime}\right) \\
& \times \sum_{\kappa=1}^{M_{q}} \frac{1}{\left\{\Delta_{\kappa}^{(q)}\left(\nu_{1}, \ldots, \nu_{n}, \nu_{1}^{\prime}, \ldots, \nu_{m}^{\prime}\right)\right\}^{m+\alpha^{\prime}}} .
\end{aligned}
$$

Prenons un intervalle quelconque $I_{q \nu_{1}}^{(1)} \times \ldots \times I_{q \nu_{n}}^{(n)}$ dans $E_{n} ;$ soit $I_{q-1, \pi_{1}}^{(1)} \times$ $\ldots \times I_{q-1, \pi_{n}}^{(n)}$ l'intervalle qui le contient. Nous considérons les $3^{n}-1$ intervalles $\left\{I_{q-1, p_{1}}^{(1)} \times \ldots \times I_{q-1, p_{n}}^{(n)}\right\}$ qui sont les voisins de $I_{q-1, \pi_{1}}^{(1)} \times \ldots \times I_{q-1, \pi_{n}}^{(n)}$. Si deux de ces $3^{n}$ cubes sont en face l'un de l'autre, nous faisons coïncider par translation les couches de profondeur $\lambda_{q}$, placées vis à vis et situées respectivement dans ces deux cubes; on obtient ainsi un intervalle de hauteur $2 \lambda_{q-1}-\lambda_{q}$. On superpose de la même manière tous les couples de cubes qui se font face. Ainsi on obtient un cube $A_{1}$ de côté $3 \lambda_{q-1}-2 \lambda_{q}$ qui contient dans sa partie centrale le cube $I_{q-1, \pi_{1}}^{(1)} \times \ldots \times I_{q-1, \pi_{n}}^{(n)}$. Désignons par $f(Q)$ la transformation qui coïncide sur chacun des $3^{n}$ cubes ci-dessus avec la translation indiquée; $f(\boldsymbol{Q})=\boldsymbol{Q}$ pour $Q \in I_{q-1, \pi_{1}}^{(1)} \times \ldots \times I_{q-1, \pi_{n}}^{(n)}$. Le cube $A_{1}$ contient $\left(3 k_{q}-2\right)^{n}$ cubes de $\left\{I_{q \jmath_{1}}^{(1)} \times \ldots \times I_{q \rho_{n}}^{(n)}\right\}$ et leurs translatés.

Cela étant, soit $S_{q 0}^{(q)}=S_{q 0}^{(q)}\left(\nu_{1}, \ldots, \nu_{n}\right)$ la surface du cube de côté $\delta_{q}+\lambda_{q}$ dont les côtés sont à distance $\delta_{r} / 2$ de $I_{q \nu_{1}}^{(1)} \times \ldots \times I_{q \nu_{n}}^{(n)}$ et pareils aux côtés de celui-ci, soit $A_{q 1}^{(q)}=A_{q 1}^{(q)}\left(\nu_{1}, \ldots, \nu_{n}\right)$ le cube fermé de côte $3\left(\delta_{q}+\lambda_{q}\right)$ dont les cêtés sont à distance $\delta_{q} / 2+\left(\delta_{q}+\lambda_{q}\right)$ de $I_{q \nu_{1}}^{(1)} \times \ldots \times I_{q \nu_{n}}^{(n)}$ et pareils aux côtés de celui-ci, soit $S_{q 1}^{(q)}=S_{q 1}^{(q)}\left(\nu_{1}, \ldots, \nu_{n}\right)$ sa surface, et ainsi de suite. Pour tout $k \geqq 2 k_{q}, S_{q k}^{(q)}$ contient $\mathrm{A}_{1}$ strictement dans son intérieur. Nous employons la même notation $S_{q k}^{(q)}$ pour l'image réciproque de $S_{q k}^{(q)}$ par $f(Q)$. Alors $S_{q k}^{(q)}=\phi$ pour $k \gtrsim 2 k_{q}$. A partir de $I_{q-1, \pi_{1}}^{(1)} \times \ldots \times I_{q-1, \pi_{n}}^{(n)}$, nous faisons le même procédé et définissons $A_{q-1, k}^{(q)}$ et $S_{q-1, k}^{(q)}$, ensuite $A_{q-2, k}^{(q)}$ et $S_{q-2, k}^{(q)}$ jusqu'à $A_{1 k}^{(q)}$ et $S_{1 k}^{(q)}$. Nous définissons $A_{i k}^{(q) \prime}$ et $S_{i k}^{(q) \prime}, 1 \leqq i \leqq q$, de la même manière dans $E_{m}$.

4. 4. Choisissons maintenant une série $k_{1}, k_{1}^{\prime}, k_{2}, k_{2}^{\prime}, \ldots$ rapidement croissante ; prenons, par exemple, $k_{1}=2, k_{1}^{\prime}=k_{1}^{k_{1}}, k_{2}=k_{1}^{\prime k_{1}^{\prime}}, \ldots$, et posons $p_{q}=k_{q}^{(n / \alpha)-1}, p_{q}^{\prime}=k_{q}^{\prime(m / 3)-19)}$ Alors

$$
\lambda_{q}=\frac{1}{\left(k_{1} \cdots k_{q}\right)^{n / \alpha}}, \quad \lambda_{q}^{\prime}=\frac{1}{\left(k_{1}^{\prime} \cdot \cdots k_{q}^{\prime}\right)^{m / / 3}},
$$

et

9: C'est l'exemple donné par Besicovitch-Moran [4] dans le cas $n=m=1$. 


$$
\begin{aligned}
& \frac{1}{\left(k_{1} \cdots k_{q-1}\right)^{n / \alpha} k_{q}} \leqq \delta_{q}+\lambda_{q} \leqq \frac{2}{\left(k_{1} \cdots k_{q-1}\right)^{n / \alpha} k_{q}}, \\
& \frac{1}{\left(k_{1}^{\prime} \cdots k_{q-1}^{\prime}\right)^{m / 3} k_{q}^{\prime}} \leqq \delta_{q}^{\prime}+\lambda_{q}^{\prime} \leqq \frac{2}{\left(k_{1}^{\prime} \cdots k_{q-1}^{\prime}\right)^{m / s} k_{q}^{\prime}} \cdot
\end{aligned}
$$

Comme

$$
\sum_{q=1}^{\infty} \frac{\left(p_{1} \cdots p_{q}\right)^{\alpha}}{\left(k_{1} \cdots k_{q}\right)^{n-\alpha}}=\sum_{q=1}^{\infty} 1=\infty \quad\left(\sum_{q=1}^{\infty} \frac{\left(p_{1}^{\prime} \cdots p_{q}^{\prime}\right)^{\beta}}{\left(k_{1}^{\prime} \cdots k_{q}^{\prime}\right)^{m-\beta}}=\infty \quad \text { resp. }\right)
$$

et

$$
\begin{aligned}
& \sum_{q=1}^{\infty} \frac{\left(p_{1} \cdots p_{q}\right)^{\alpha_{1}}}{\left(k_{1} \cdots k_{q}\right)^{n-\alpha_{1}}}=\sum_{q=1}^{\infty} \frac{1}{\left(k_{1} \cdots k_{q}\right)^{n-\alpha_{1}-((n / \alpha)-1) \alpha_{1}}} \\
& \quad=\sum_{q=1}^{\infty} \frac{1}{\left(k_{1} \cdots k_{q}\right)^{n\left(1-\alpha_{1} / \alpha\right)}}<\infty \quad\left(\sum_{q=1}^{\infty} \frac{\left(p_{1}^{\prime} \cdots p_{q}^{\prime}\right)^{\beta_{1}}}{\left(k_{1}^{\prime} \cdots k_{q}^{\prime}\right)^{m-\beta_{1}}}<\infty \quad \text { resp. }\right)
\end{aligned}
$$

pour $\alpha_{1}, 0<\alpha_{1}<\alpha$, quelconque (pour $\beta_{1}, 0<\beta_{1}<\beta$, quelconque resp.), la dimension de $F$ ( $F^{\prime}$ resp.) est égale à $\alpha$ ( $\beta$ resp.).

Nous allons voir que la capacité d'ordre $m+\alpha^{\prime}$ de $F \times F^{\prime}$ est positive. D'abord nous déterminons un entier $r_{q}$ tel que

$$
\begin{aligned}
\frac{\delta_{q}^{\prime}}{2}<\delta_{q}^{\prime}+\lambda_{q}^{\prime}<2\left(\delta_{q}^{\prime}+\lambda_{q}^{\prime}\right)<\ldots & <r_{q}\left(\delta_{q}^{\prime}+\lambda_{q}^{\prime}\right) \\
& \leqq \frac{\delta_{q}}{2}<\left(r_{q}+1\right)\left(\delta_{q}^{\prime}+\lambda_{q}^{\prime}\right)<\ldots
\end{aligned}
$$

Pour les $\left\{P_{\kappa}^{(q)}\right\}$ contenus dans le produit de $S_{q 0}^{(q)}=S_{q 0}^{(q)}\left(\nu_{1}, \ldots, \nu_{n}\right)$ et $A_{q r_{q}}^{(q)}$, $=A_{q r_{q}}^{(q) \prime}\left(\nu_{i}^{\prime}, \ldots, \nu_{m}^{\prime}\right)$, on pose

$$
\Delta_{\kappa}^{(q)}=\frac{\delta_{q}}{2}
$$

Pour ces points on fait la somme

$$
\begin{aligned}
\sum_{q 0}^{(1)} & =\sum \frac{1}{\left(\Delta_{\kappa}^{(q)}\right)^{m+\alpha^{\prime}}}=2^{n}\left\{2\left(r_{q}+1\right)\right\}^{m}\left(\frac{2}{\delta_{q}}\right)^{m+\alpha^{\prime}}=2^{n+m}\left(\frac{r_{q}+1}{r_{q}}\right)^{m} r_{q}^{m}\left(\frac{2}{\delta_{q}}\right)^{m+\alpha^{\prime}} \\
& \leqq 2^{n+2 m} \frac{1}{\left(\delta_{q}^{\prime}+\lambda_{q}^{\prime}\right)^{m+\alpha^{\prime}}}\left(\frac{2}{\delta_{q}}\right)^{\alpha^{\prime}}=\frac{2^{n+2 m+\alpha^{\prime}}}{\left(\delta_{q}^{\prime}+\lambda_{q}^{\prime}\right)^{m+\alpha^{\prime}} \delta_{q}^{\alpha^{\prime}}} .
\end{aligned}
$$

Pour les autres $\left\{P_{\kappa}^{(q)}\right\}$ contenus dans $S_{q 0}^{(q)} \times A_{q, 2 q^{\prime}}^{(q) \prime}$, on pose

$$
\Delta_{\kappa}^{(q)}=\left(r_{q}+1\right)\left(\delta_{q}^{\prime}+\lambda_{q}^{\prime}\right),\left(r_{q}+2\right)\left(\delta_{q}^{\prime}+\lambda_{q}^{\prime}\right), \ldots
$$

Pour ces points on fait la somme 


$$
\begin{aligned}
\sum_{q 0}^{(2)} & =\sum \frac{1}{\left(\Delta_{\kappa}^{(q)}\right)^{m+\alpha^{\prime}}} \leqq 2^{n} \frac{\left\{2\left(r_{q}+2\right)\right\}^{m}-\left\{2\left(r_{q}+1\right)\right\}^{m}}{\left\{\left(r_{q}+1\right)\left(\delta_{q}^{\prime}+\lambda_{q}^{\prime}\right)\right\}^{m+\alpha^{\prime}}} \\
& +2^{n} \frac{\left\{2\left(r_{q}+3\right)\right\}^{m}-\left\{2\left(r_{q}+2\right)\right\}^{m}}{\left\{\left(r_{q}+2\right)\left(\delta_{q}^{\prime}+\lambda_{q}^{\prime}\right)\right\}^{m+\alpha^{\prime}}}+\ldots+2^{n} \frac{\left\{2\left(2 k_{q}^{\prime}+1\right)\right\}^{m}-\left(4 k_{q}^{\prime}\right)^{m}}{\left\{2 k_{q}^{\prime}\left(\delta_{q}^{\prime}+\lambda_{q}^{\prime}\right)\right\}^{m+\alpha^{\prime}}} .
\end{aligned}
$$

Nous notons que $(s+1)^{m}-s^{m} \leqq\left(2^{m}-1\right) s^{m-1}$ pour $s \geqq 1$, et obtenons

$$
\begin{aligned}
\sum_{q 0}^{(2)} & \leqq \frac{2^{n+m}\left(2^{m}-1\right)}{\left(\delta_{q}^{\prime}+\lambda_{q}^{\prime}\right)^{m+\alpha^{\prime}}} \sum_{s=r_{q+1}}^{2 k_{q^{\prime}}}-\frac{1}{s^{\alpha^{\prime}+1}} \leqq \frac{2^{n+2 m}}{\left(\delta_{q}^{\prime}+\lambda_{q}^{\prime}\right)^{m+\alpha^{\prime}}} \frac{1}{\alpha^{\prime}}\left(\frac{1}{r_{q}^{\alpha^{\prime}}}-\frac{1}{\left(2 k_{q}^{\prime}\right)^{\alpha^{\prime}}}\right) \\
& <\frac{2^{n+2 m}}{\alpha^{\prime}\left(\delta_{q}^{\prime}+\lambda_{q}^{\prime}\right)^{m+\alpha^{\prime}}}\left(\frac{r_{q}+1}{r_{q}}\right)^{\alpha^{\prime}}\left(\frac{\delta_{q}^{\prime}+\lambda_{q}^{\prime}}{\delta_{q} / 2}\right)^{\alpha^{\prime}}<\frac{2^{n+2 m+2 \alpha^{\prime}}}{\alpha^{\prime}\left(\delta_{q}^{\prime}+\lambda_{q}^{\prime}\right)^{m} \delta_{q}^{\alpha^{\prime}}}
\end{aligned}
$$

Si on pose $\sum_{q 0}=\sum_{q 0}^{(1)}+\sum_{q 0}^{(2)}$, on aura

$$
\sum_{q 0}<\frac{c}{\left(\delta_{q}^{\prime}+\lambda_{q}^{\prime}\right)^{m} \delta_{q}^{\alpha^{\prime}}}
$$

avec une constante $c=2^{n+2 m+2 \alpha^{\prime}} / \alpha^{\prime}$.

Ensuite nous déterminons un entier $t_{q}$ tel que

$$
\ldots<\left(r_{q}+1\right)\left(\delta_{q}^{\prime}+\lambda_{q}^{\prime}\right)<\ldots<t_{q}\left(\delta_{q}^{\prime}+\lambda_{q}^{\prime}\right) \leqq \lambda_{q}+\delta_{q}<\left(t_{q}+1\right)\left(\delta_{q}^{\prime}+\lambda_{q}^{\prime}\right)<\ldots
$$

Pour tous $\left\{P_{\mathrm{k}}^{(q)}\right\}$ contenus dans le produit de $S_{q 1}^{(q)}=S_{q 1}^{(q)}\left(\nu_{1}, \ldots, \nu_{n}\right)$ et de $A_{q t_{q}}^{(q) \prime}$ $=A_{q t_{q}}^{(q) \prime}\left(\nu_{1}^{\prime}, \ldots, \nu_{m}^{\prime}\right)$, nous posons

$$
\Delta_{\kappa}^{(q)}=\delta_{q}+\lambda_{q},
$$

et, pour les autres $\left\{P_{\kappa}^{(q)}\right\}$ contenus dans $S_{q 1}^{(q)} \times A_{q, 2 k q^{\prime}}^{(q) \prime}$, nous posons

$$
\Delta_{\kappa}^{(q)}=\left(t_{q}+1\right)\left(\delta_{q}^{\prime}+\lambda_{q}^{\prime}\right),\left(t_{q}+2\right)\left(\delta_{q}^{\prime}+\lambda_{q}^{\prime}\right), \ldots
$$

Pour tous ces points, nous faisons la somme

$$
\begin{aligned}
\sum_{q 1}= & \sum \frac{1}{\left(\Delta_{\kappa}^{(q)}\right)^{m+\alpha^{\prime}}} \leqq\left\{(2 \cdot 2)^{n}-(2 \cdot 1)^{n}\right\} \frac{\left\{2\left(t_{q}+1\right)\right\}^{m}}{\left(\delta_{q}+\lambda_{q}\right)^{m+\alpha^{\prime}}} \\
& +\left\{(2 \cdot 2)^{n}-(2 \cdot 1)^{n}\right\} 2^{m} \sum_{s=t_{q+1}}^{2 k_{q^{\prime}}} \frac{(s+1)^{m}-s^{m}}{\left\{s\left(\delta_{q}^{\prime}+\lambda_{q}^{\prime}\right)\right\}^{m+\alpha^{\prime}}} \\
\leqq & 2^{n+m}\left(2^{n}-1\right)\left\{\frac{\left(t_{q}+1\right)^{m}}{\left(\delta_{q}+\lambda_{q}\right)^{m+\alpha^{\prime}}}+\frac{2^{m}}{\left(\delta_{q}^{\prime}+\lambda_{q}^{\prime}\right)^{m+\alpha^{\prime}}} \sum_{s=t_{q+1}}^{2 k_{q^{\prime}}}-\frac{1}{s^{\alpha^{\prime}+1}}\right\} \\
< & \frac{2 c\left(2^{n}-1\right)}{\left(\delta_{q}^{\prime}+\lambda_{q}^{\prime}\right)^{m}\left(\delta_{q}+\lambda_{q}\right)^{\alpha^{\prime}}} .
\end{aligned}
$$

Soit $u_{q}$ le plus petit entier tel que $2 k_{q}^{\prime}\left(\delta_{q}^{\prime}+\lambda_{q}^{\prime}\right) \leqq u_{q}\left(\delta_{q}+\lambda_{q}\right)$. Pour les points $\left\{P_{\kappa}^{(q)}\right\}$ contenus dans $A_{q u_{q}}^{(q)} \times A_{q, 2 k_{q^{\prime}}}^{(q){ }^{\prime}}$, on a 


$$
\begin{aligned}
& \Sigma_{q}=\sum_{q 0}+\sum_{q 1}+\ldots=\frac{c}{\left(\delta_{q}^{\prime}+\lambda_{q}^{\prime}\right)^{m} \delta_{q}^{\alpha^{\prime}}}+\frac{2 c\left(2^{n}-1\right)}{\left(\delta_{q}^{\prime}+\lambda_{q}^{\prime}\right)^{m}\left(\delta_{q}+\lambda_{q}\right)^{\alpha^{\prime}}} \\
& +\frac{2 c\left(3^{n}-2^{n}\right)}{\left(\delta_{q}^{\prime}+\lambda_{q}^{\prime}\right)^{m} 2^{\alpha^{\prime}}\left(\delta_{q}+\lambda_{q}\right)^{\alpha^{\prime}}}+\ldots+\frac{2 c\left\{\left(u_{q}+1\right)^{n}-u_{q}^{n}\right\}}{\left(\delta_{q}^{\prime}+\lambda_{q}^{\prime}\right)^{m} u_{q}^{\alpha^{\prime}}\left(\delta_{q}+\lambda_{q}\right)^{\alpha^{\prime}}} \\
& \leqq \frac{c}{\left(\delta_{q}^{\prime}+\lambda_{q}^{\prime}\right)^{m} \delta_{q}^{\alpha^{\prime}}}+\frac{c 2^{n+1}}{n-\alpha^{\prime}} \frac{u_{q}^{n-\alpha^{\prime}}}{\left(\delta_{q}^{\prime}+\lambda_{q}^{\prime}\right)^{m}\left(\delta_{q}+\lambda_{q}\right)^{\alpha^{\prime}}} \\
& \left.\leqq \delta_{q}^{\prime}+\lambda_{q}^{\prime}\right)^{m} \delta_{q}^{\alpha^{\prime}}+\underset{n-\alpha^{\prime}}{c}+2^{4 n-2 \alpha^{\prime}+1} \frac{k_{q}^{\prime n-\alpha^{\prime}}}{\left(\delta_{q}^{\prime}+\lambda_{q}^{\prime}\right)^{m-n+\alpha^{\prime}}\left(\delta_{q}+\lambda_{q}\right)^{n}} \\
& \leqq c\left(k_{1}^{\prime} \cdots k_{q-1}^{\prime}\right)^{m^{2 / \beta}} k_{q}^{\prime m}\left\{\frac{k_{q}^{n / \alpha-1}-1}{k_{1} \cdots k_{q-1}\left(k_{q}-1\right)\left(k_{1} \cdot \cdots k_{q}\right)^{n / \alpha-1}}\right\}^{-\alpha^{\prime}} \\
& +\frac{c 2^{4 n-2 \alpha^{\prime}+1}}{n-\alpha^{\prime}} k_{q}^{\prime n-\alpha^{\prime}}\left(k_{1}^{\prime} \cdots k_{q-1}^{\prime}\right)^{m / \beta\left(m-n+\alpha^{\prime}\right)} k_{q}^{\prime m-n+\alpha^{\prime}}\left(k_{1} \cdots k_{q-1}\right)^{n^{2} / \alpha} k_{q}^{n} \\
& \leqq c_{1}\left(k_{1}^{\prime} \cdots k_{q-1}^{\prime}\right)^{m^{2} / \beta} k_{q}^{\prime m}\left(k_{1} \cdots k_{q-1}\right)^{n \alpha^{\prime} / \alpha} k_{q}^{\alpha^{\prime}} \\
& +c_{1}\left(k_{1}^{\prime} \cdots k_{q-1}^{\prime}\right)^{m / 3\left(m-n+\alpha^{\prime}\right)} k_{q}^{\prime m}\left(k_{1} \cdots k_{q-1}\right)^{n^{2} / \alpha} k_{q}^{n}
\end{aligned}
$$

où

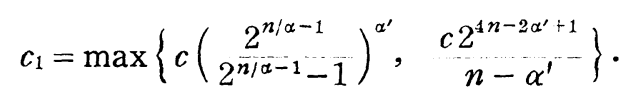

Puis, pour les points $\left\{P_{\kappa}^{(q)}\right\}$ contenus dans $\left(A_{q, 3 k_{q}}^{(q)}-A_{q u_{q}}^{(q)}\right) \times A_{q, 2 k_{q^{\prime}}}^{(q) \prime}$, on a

$$
\begin{aligned}
\Sigma_{q}^{\prime} & =\sum \frac{1}{\left(\Delta_{\kappa}^{(q)}\right)^{m+\alpha^{\prime}}} \leqq \frac{\left(2 k_{q}^{\prime}\right)^{m} 2^{n}}{\left(u_{q}\left(\delta_{q}+\lambda_{q}\right)\right)^{m+\alpha^{\prime}}}\left\{\left(2 u_{q}+1\right)^{n}-\left(u_{q}+1\right)^{n}\right. \\
& \left.+\frac{\left(3 u_{q}+1\right)^{n}-\left(2 u_{q}+1\right)^{n}}{2^{m+\alpha^{\prime}}}+\ldots+\frac{\left(v_{q} u_{q}+1\right)^{n}-\left(\left(v_{q}-1\right) u_{q}+1\right)^{n}}{\left(v_{q}-1\right)^{m+\alpha^{\prime}}}\right\},
\end{aligned}
$$

où $v_{q}$ est le plus petit entier tel que $v_{q} u_{q} \geqslant 2 k_{q}$. Il vient que

$$
\begin{aligned}
\sum_{q}^{\prime} & \leqq \frac{\left(2 k_{q}^{\prime}\right)^{m} 2^{n}}{\left(u_{1}\left(\delta_{q}+\lambda_{q}\right)\right)^{m+\alpha^{\prime}}} \sum_{s=1}^{v_{q-1}} \frac{\left\{(s+1) u_{q}+1\right\}^{n}-\left(s u_{q}+1\right)^{n}}{s^{m+\alpha^{\prime}}} \\
& \leqq \frac{\left(2 k_{q}^{\prime}\right)^{m} 2^{n} u_{q}^{n}}{\left(u_{q}\left(\delta_{q}+\lambda_{q}\right)\right)^{m+\alpha^{\prime}}} \sum_{s=1}^{v_{q}-1}(s+2)^{n}-(s+1)^{n} \\
s^{m+\alpha^{\prime}} & \leqq \frac{\left(2 k_{q}^{\prime}\right)^{m} 2^{n} u_{q}^{n-m-\alpha^{\prime}}}{\left(\delta_{q}+\lambda_{q}\right)^{m+\alpha^{\prime}}} 2^{2 n-1} \sum_{s=1}^{v_{q}-1} s^{n-1-m-\alpha^{\prime}} .
\end{aligned}
$$

On considère les trois cas: $n-m-\alpha^{\prime}>0,=0,<0$. Nous aurons respectivement

$$
\sum_{s=1}^{v_{q-1}} s^{n-1-m-\alpha^{\prime}} \leqq 1+\frac{1}{n-m-\alpha^{\prime}}, \quad 1+\log v_{q}, \quad \begin{gathered}
1 \\
m+\alpha^{\prime}-n
\end{gathered}
$$

Dans le premier cas 


$$
\begin{aligned}
\Sigma_{q}^{\prime} & \leqq\left(1+\frac{1}{n-m-\alpha^{\prime}}\right) \frac{2^{3 n+m-1} k_{q}^{\prime m} u_{q}^{n-m-\alpha^{\prime}} v_{q}^{n-m-\alpha^{\prime}}}{\left(\delta_{q}+\lambda_{q}\right)^{m+\alpha^{\prime}}} \\
& \leqq\left(1+\frac{1}{n-m-\alpha^{\prime}}\right) \frac{2^{3 n+m-1} k_{q}^{\prime m} 2^{n-m-\alpha^{\prime}}\left(2 k_{q}\right)^{n-m-\alpha^{\prime}}}{\left(\delta_{q}+\lambda_{q}\right)^{m+\alpha^{\prime}}} \\
& \leqq\left(1+\frac{1}{\left.n-\frac{\alpha^{\prime}}{m-\alpha^{\prime}}\right) 2^{5 n-m-2 \alpha^{\prime}-1} k_{q}^{\prime m}\left(k_{1} \cdots k_{q-1}\right)^{n / \alpha\left(m+\alpha^{\prime}\right)} k_{q}^{n} .}\right. \\
& \leqq 2^{5 n-m-2 \alpha^{\prime}-1}\left(1+\frac{1}{n-m-\alpha^{\prime}}\right)\left(k_{1} \cdots k_{q-1}\right)^{n^{2} / \alpha} k_{q}^{n} k_{q}^{\prime m} .
\end{aligned}
$$

Dans le deuxième cas on peut supposer que $\log v_{q} \geqslant 1$ et on obtient

$$
\begin{aligned}
\sum_{q}^{\prime} & \leqq \frac{2^{m+3 n} k_{q}^{\prime m}}{\left(\delta_{q}+\lambda_{q}\right)^{n}} \log v_{q} \leqq \frac{2^{m+3 n} k_{q}^{\prime m}}{\left(\delta_{q}+\lambda_{q}\right)^{n}} \log \frac{4 k_{q}}{u_{q}} \leqq \frac{2^{m+3 n} k_{q}^{\prime m}}{\left(\delta_{q}+\lambda_{q}\right)^{n}} \log \frac{4 k_{q}\left(\delta_{q}+\lambda_{q}\right)}{2 k_{q}^{\prime}\left(\delta_{q}^{\prime}+\lambda_{q}^{\prime}\right)} \\
& \leqq 2^{m+3 n} k_{q}^{\prime m}\left(k_{1} \cdots k_{q-1}\right)^{n^{2 / \alpha}} k_{q}^{n} \log \frac{2\left(k_{1}^{\prime} \cdots k_{q-1}^{\prime}\right)^{m / 3}}{\left(k_{1} \cdots k_{q-1}\right)^{n / \alpha}} .
\end{aligned}
$$

Dans le troisième cas

$$
\begin{aligned}
\Sigma_{q}^{\prime} & \leqq \frac{2^{m+3 n-1}}{m+\alpha^{\prime}-n} \frac{1}{u_{q}^{m+\alpha^{\prime}-n}\left(\delta_{q}+\lambda_{q}\right)^{m+\alpha^{\prime}}} \\
& \leqq \frac{2^{m+3 n-1}}{m+\alpha^{\prime}-n} \frac{1}{\left(\delta_{q}+\lambda_{q}\right)^{n}\left\{2 k_{q}^{\prime}\left(\delta_{q}^{\prime}+\lambda_{q}^{\prime}\right)\right\}^{m+\alpha^{\prime}-n}} \\
& \leqq \frac{2^{4 n-1-\alpha^{\prime}}}{m+\alpha^{\prime}-n}\left(k_{1}^{\prime} \cdots k_{q-1}^{\prime}\right)^{m / \beta\left(m+\alpha^{\prime}-n\right)}\left(k_{1} \cdots k_{q-1}\right)^{n^{2} / \alpha} k_{q}^{n}
\end{aligned}
$$

La somme $\sum 1 /\left(\Delta_{\kappa}^{(q)}\right)^{m+\alpha^{\prime}}$ pour tous les $\left\{P_{\kappa}^{(q)}\right\}$ contenus dans $A_{q, 2 k_{q}}^{(q)} \times A_{q, 2 q^{\prime}}^{(q) \prime}$ est égale à $\Sigma_{q}+\Sigma_{q}^{\prime}$.

4. 5. Nous continuerons et terminerons l'évaluation. Soit $w_{q}$ le plus petit entier tel que $\delta_{q-1}^{\prime}+\lambda_{q-1}^{\prime} \leqq w_{q}\left(\delta_{q}+\lambda_{q}\right), x_{q}$ le plus petit entier tel que $2\left(\delta_{q-1}+\lambda_{q-1}\right)$ $\leqq x_{q}\left(\delta_{q}+\lambda_{q}\right)$, ainsi de suite et enfin $u_{q}^{\prime}$ le plus petit entier tel que $2 k_{q}\left(\delta_{q}+\lambda_{q}\right)$ $\leqq u_{q}^{\prime}\left(\delta_{q-1}^{\prime}+\lambda_{q-1}^{\prime}\right)$. Pour les points $\left\{P_{\kappa}^{(q)}\right\}$ contenus dans $A_{q, 2 k_{q}}^{(q)} \times\left(A_{q-1,2}^{(q) \prime}-A_{q-1,1}^{(q)}\right)$, nous posons

et obtenons

$$
\Delta_{\mathrm{x}}^{(q)}=\delta_{q-1}^{\prime}+\lambda_{q-1}^{\prime}, \quad 2\left(\delta_{q-1}^{\prime}+\lambda_{q-1}^{\prime}\right), \ldots
$$

$$
\begin{aligned}
\sum \frac{1}{\left(\Delta_{\kappa}^{(q)}\right)^{m+\alpha^{\prime}}} \leqq & 2^{m}\left(3^{m}-2^{m}\right)\left\{\frac{k_{q}^{\prime m} w_{q}^{n}}{\left(\delta_{q-1}^{\prime}+\lambda_{q-1}^{\prime}\right)^{m+\alpha^{\prime}}}+\frac{k_{q}^{\prime}\left(2^{n}-1^{n}\right) w_{q}^{n}}{2^{m+\alpha^{\prime}}\left(\delta_{q-1}^{\prime}+\lambda_{q-1}^{\prime}\right)^{m+\alpha^{\prime}}}\right. \\
& +\ldots+\frac{k_{q}^{\prime}\left(\left(u_{q}^{\prime}+1\right)^{n}-u_{q}^{\prime}\right) w_{q}^{n}}{\left.u_{q}^{\prime m+\alpha^{\prime}}\left(\delta_{q-1}^{\prime}+\lambda_{q-1}^{\prime}\right)^{m+\alpha^{\prime}}\right\}} \\
= & \frac{2^{m}\left(3^{m}-2^{m}\right) k_{q}^{\prime m} w_{q}^{m}}{\left(\delta_{q-1}^{\prime}+\lambda_{q-1}^{\prime}\right)^{m+\alpha^{\prime}}}\left\{1+\sum_{s=1}^{u_{q^{\prime}}} \frac{(s+1)^{n}-s^{n}}{s^{m+\alpha^{\prime}}}\right\} \\
\leqq & \frac{2^{m}\left(3^{m}-2^{m}\right) k_{q}^{\prime m} w_{q}^{m}}{\left(\delta_{q-1}^{\prime}+\lambda_{q-1}^{\prime}\right)^{m+\alpha^{\prime}}}\left(1+2^{n} \sum_{s=1}^{u_{q^{\prime}}^{\prime}} s^{n-1-m-\alpha^{\prime}}\right) .
\end{aligned}
$$


Pour les points $\left\{P_{\kappa}^{(q)}\right\}$ contenus ḋans $A_{q, 2 k_{q}}^{(q)} \times\left(A_{q-1,2}^{(q)}-A_{q-1,2}^{(q)}\right)$ on aura

$$
\sum \frac{1}{\left(\Delta_{\kappa}^{(q)}\right)^{m+\alpha^{\prime}}} \leqq \begin{gathered}
2^{m}\left(4^{m}-3^{m}\right) k_{q}^{\prime m} w_{q}^{m} \\
\left(\delta_{q-1}^{\prime}+\lambda_{q-1}^{\prime}\right)^{m+\alpha^{\prime}}
\end{gathered}\left\{\frac{2^{n}}{2^{n+\alpha^{\prime}}}+2^{n} \sum_{s=2}^{n u_{q^{\prime}}} s^{n-1-m-\alpha^{\prime}}\right\} .
$$

Et ainsi de suite.

Pour tous les $\left\{P_{\kappa}^{(q)}\right\}$ contenus dans $A_{q, 2 k_{q}}^{(q)} \times\left(A_{q-1, u_{q}^{\prime}+1}^{(q)}-A_{q-1,1}^{(q) \prime}\right)$ nous aurons

$$
\begin{aligned}
& \sum_{q-1}^{*}=\sum \frac{1}{\left(\Delta_{*}^{(q)}\right)^{m+\alpha^{\prime}}} \\
& \leqq \frac{2^{m} k_{q}^{\prime m} w_{q}^{m}}{\left(\delta_{q-1}^{\prime}+\lambda_{q-1}^{\prime}\right)^{m+\alpha^{\prime}}}\left[\left\{\left(3^{n n}-2^{m}\right)+\left(4^{m}-3^{m}\right) 2^{m-m-\alpha^{\prime}}+\ldots\right.\right. \\
& \left.+\left(\left(u_{q}^{\prime}+2\right)^{m}-\left(\boldsymbol{u}_{q}^{\prime}+1\right)^{m}\right) \boldsymbol{u}_{q}^{\prime n-m-\alpha^{\prime}}\right\}+\left\{\left(3^{m}-2^{m}\right) \sum_{s=1}^{u q^{\prime}}\right. \\
& \left.\left.+\left(4^{m}-3^{m}\right) \sum_{s=2}^{u_{q^{\prime}}}+\ldots+\left(\left(u_{q}^{\prime}+2\right)^{m}-\left(u_{q}^{\prime}+1\right)^{m}\right) \sum_{s=u_{q^{\prime}}}^{u_{q^{\prime}}}\right\} s^{n-1-m-\alpha^{\prime}}\right] \\
& =\frac{2^{m} k_{q}^{\prime} w_{q}^{n}}{\left(\delta_{q-1}^{\prime}+\lambda_{q-1}^{\prime}\right)^{m+\alpha^{\prime}}}\left\{2^{m} \sum_{s=1}^{u_{q^{\prime}}}(s+1)^{m-1} s^{n-m-x^{\prime}}+\sum_{s=1}^{u_{q^{\prime}}^{\prime}}(s+2)^{m} s^{n-1-m-\alpha^{\prime}}\right. \\
& \left.+\left(u_{q}^{\prime}+2\right)^{m} u_{q}^{\prime n-1-m-\alpha^{\prime}}\right\}=\frac{2^{3 m+n-\alpha^{\prime}+2} k_{q}^{\prime m} w_{q}^{n} u_{q}^{\prime n-\alpha^{\prime}}}{\left(n-\alpha^{\prime}\right)\left(\delta_{q-1}^{\prime}+\lambda_{q-1}^{\prime}\right)^{m+\alpha^{\prime}}} \\
& \leqq \frac{2^{4 m+3 n-3 \alpha^{\prime}+2} k_{q}^{\prime m} k_{q}^{n-\alpha^{\prime}}}{\left(n-\alpha^{\prime}\right)\left(\delta_{q}+\lambda_{q}\right)^{\alpha^{\prime}}\left(\delta_{q-1}^{\prime}+\lambda_{q-1}^{\prime}\right)^{m}} \\
& \leqq c_{2}\left(k_{1} \cdots k_{q-1}\right)^{n \alpha^{\prime} / \alpha} k_{q}^{n}\left(k_{1}^{\prime} \cdots k_{q-2}^{\prime}\right)^{m^{2} / 3} k_{q-1}^{\prime m} k_{q}^{\prime m}
\end{aligned}
$$

avec $c_{2}=2^{4 m+3 n-3 \alpha^{\prime}+2} /\left(n-\alpha^{\prime}\right)$ pour assez grand $q$.

Pour les points $\left\{P_{\kappa}^{(q)}\right\}$ contenus dans $A_{q, 2 k_{q}}^{(q)} \times\left(A_{q-1,2 k^{\prime} q-1}^{(q)}-A_{q-1, u q^{\prime}+1}^{(q) \prime}\right)$ on a la somme

$$
\begin{aligned}
& \sum_{q-1}^{* \prime}=\sum \underset{\left(\Delta_{\kappa}^{(q)}\right)^{m+\alpha^{\prime}}}{1} \leqq \frac{\left(2 k_{q}\right)^{n} k_{q}^{\prime m}}{\left(u_{q}^{\prime}\left(\delta_{q-1}^{\prime}+\lambda_{q-1}^{\prime}\right)\right)^{m+\alpha^{\prime}}}\left\{\left(2\left(u_{q}^{\prime}+1\right)+1\right)^{m}-\left(u_{q}^{\prime}+2\right)^{m}\right. \\
& +\frac{\left(3\left(u_{q}^{\prime}+1\right)+1\right)^{m}-\left(2\left(u_{q}^{\prime}+1\right)+1\right)^{m}}{2^{m+\alpha^{\prime}}}+\ldots \\
& \left.+\frac{\left(v_{q}^{\prime}\left(u_{q}^{\prime}+1\right)+1\right)^{m}-\left(\left(v_{q}^{\prime}-1\right)\left(u_{q}^{\prime}+1\right)+1\right)^{m}}{\left(v_{q}^{\prime}-1\right)^{m+\alpha^{\prime}}}\right\},
\end{aligned}
$$

où $v_{q-1}^{\prime}$ est le plus petit entier tel que $v_{q-1}^{\prime} u_{q-1}^{\prime} \geq 2 k_{q-1}^{\prime}$. Il vient que

$$
\begin{aligned}
& \sum_{q-1}^{* \prime} \leqq \frac{2^{n} k_{q}^{n} k_{q}^{\prime m} 2^{m} 2^{2 m}}{u_{q}^{\prime \alpha^{\prime}}\left(\delta_{q-1}^{\prime}+\lambda_{q-1}^{\prime}\right)^{m+\alpha^{\prime}}} \sum_{s=1}^{v_{q^{\prime}-1}^{\prime}} \frac{1}{s^{\alpha^{\prime}+1}} \leqq \frac{2^{n+3 m} k_{q}^{n} k_{q}^{\prime m}}{\alpha^{\prime} u_{q}^{\prime \alpha^{\prime}}\left(\delta_{q-1}^{\prime}+\lambda_{q-1}^{\prime}\right)^{m+\alpha^{\prime}}} \\
& \leqq \frac{2^{n \div 3 m} k_{q}^{n} k_{q}^{\prime m}}{\alpha^{\prime}\left(2 k_{q}\right)^{\alpha^{\prime}}\left(\delta_{q}+\lambda_{q}\right)^{\alpha^{\prime}}\left(\delta_{q-1}^{\prime}+\lambda_{q-1}^{\prime}\right)^{m}} \\
& \leqq 2^{n+3 m-\alpha^{\prime}} \bar{\alpha}^{\prime}\left(k_{1} \cdots k_{q-1}\right)^{n \alpha^{\prime} / \alpha} k_{q}^{n}\left(k_{1}^{\prime} \cdots k_{q-2}^{\prime}\right)^{m^{2 / 3}}\left(k_{q-1}^{\prime} k_{q}^{\prime}\right)^{m} .
\end{aligned}
$$


La some $\sum 1 /\left(\Delta_{\kappa}^{(q)}\right)^{m+\alpha^{\prime}}$ pour tous les $\left\{P_{\kappa}^{(q)}\right\}$ contenus dans $A_{q, 2 k_{q}}^{(q)} \times A_{q-1, u q^{\prime}+1}^{(q)}$ est égale à

$$
\sum_{q-1}^{*}+\sum_{q-1}^{* \prime} \leqq c_{3}\left(k_{1} \cdots k_{q-1}\right)^{n x^{\prime} / \alpha} k_{q}^{n}\left(k_{1}^{\prime} \cdots k_{q-1}^{\prime}\right)^{m^{2 / \beta}} k_{q-1}^{\prime m} k_{q}^{\prime m},
$$

où $c_{3}$ est une constante ne dépendant que de $n, m, \alpha, \beta$ et $\alpha^{\prime}$.

Nous continuons ce procédé. Dans le cas $m+\alpha^{\prime}<n$ on a enfin

$$
\begin{aligned}
u_{q} & \leqq c_{0}\left\{\frac{\left(k_{1}^{\prime} \cdots k_{q-2}^{\prime}\right)^{m^{2} / 3-m}}{\left(k_{1} \cdots k_{q-1}\right)^{n\left(1-\alpha^{\prime} / \alpha\right)}} \frac{k_{q-1}^{\prime m^{2} / 3-m}}{k_{q}^{n-\alpha^{\prime}}}+\frac{\left(k_{1} \cdots k_{q-1}\right)^{n^{2} / \alpha-n}}{\left(k_{1}^{\prime} \cdots k_{q-1}^{\prime}\right)^{m}}\right. \\
& \left.+\frac{\left(k_{1}^{\prime} \cdots k_{q-2}^{\prime}\right)^{m^{2} / 3-m}}{\left(k_{1} \cdots k_{q-1}\right)^{n\left(1-\alpha^{\prime} / \alpha\right)}}+\frac{\left(k_{1} \cdots k_{q-2}\right)^{n^{2} / \alpha-n}}{\left(k_{1}^{\prime} \cdots k_{q-2}^{\prime}\right)^{m}}+\frac{\left(k_{1}^{\prime} \cdots k_{q-3}^{\prime}\right)^{m^{2} / 3-m}}{\left(k_{1} \cdots k_{q-2}\right)^{n\left(1-\alpha^{\prime} / \alpha\right)}}+\cdots\right\},
\end{aligned}
$$

où $c_{0}$ est une constante qui dépend seulement de $n, m, \alpha, \beta$ et $\alpha^{\prime}$.

Dans le cas $m+\alpha^{\prime}=n$,

$$
\begin{aligned}
u_{q} & \leqq c_{0}\left\{\frac{\left(k_{1}^{\prime} \cdots k_{q-2}^{\prime}\right)^{m^{2} / \beta-m}}{\left(k_{1} \cdots k_{q-1}\right)^{n\left(1-\alpha^{\prime} / \alpha\right)}} \frac{k_{q-1}^{\prime m^{2 / \beta-m}}}{k_{q}^{n-\alpha^{\prime}}}+\frac{\left(k_{1} \cdots k_{q-1}\right)^{n^{2} / \alpha-n}}{\left(k_{1}^{\prime} \cdots k_{q-1}^{\prime}\right)^{m}} \log \left(k_{1}^{\prime} \cdots k_{q-1}^{\prime}\right)\right. \\
& +\frac{\left(k_{1}^{\prime} \cdots k_{q-2}^{\prime}\right)^{m^{2} / \beta-m}}{\left(k_{1} \cdots k_{q-1}\right)^{n\left(1-\alpha^{\prime} / \alpha\right)}}+\frac{\left(k_{1} \cdots k_{q-2}\right)^{n^{2} / \alpha-n}}{\left(k_{1}^{\prime} \cdots k_{q-2}^{\prime}\right)^{m}} \log \left(k_{1}^{\prime} \cdots k_{q-2}^{\prime}\right) \\
& \left.+\frac{\left(k_{1}^{\prime} \cdots k_{q-3}^{\prime}\right)^{m^{2} / \beta-m}}{\left(k_{1} \cdots k_{q-2}\right)^{n\left(1-\alpha^{\prime} / \alpha\right)}}+\cdots\right\}=c_{0}\left\{\frac{\left(k_{1}^{\prime} \cdots k_{q-2}^{\prime}\right)^{m^{2} / \beta-m}}{\left(k_{1} \cdots k_{q-1}\right)^{n\left(1-\alpha^{\prime} / \alpha\right)}} \frac{\dot{k}_{q-1}^{\prime} m^{2 / \beta-m}}{k_{q}^{n-\alpha^{\prime}}}\right. \\
& +\sum^{q-1}\left(k_{1} \cdots k_{i}\right)^{n^{2} / \alpha-n} \\
\left(k_{1}^{\prime} \cdots k_{i}^{\prime}\right)^{m} & \left.\log \left(k_{1}^{\prime} \cdots k_{i}^{\prime}\right)+\sum^{g-1} \frac{\left(k_{1}^{\prime} \cdots k_{i-1}^{\prime}\right)^{m^{2} / \beta-m}}{\left(k_{1} \cdots k_{i}\right)^{n\left(1-\alpha^{\prime} / \alpha\right)}}\right\} .
\end{aligned}
$$

Le premier cas revient à ce cas.

Dans le cas $m+\alpha^{\prime}>n$,

$$
\begin{aligned}
u_{q} & \leqq c_{0}\left\{\frac{\left(k_{1}^{\prime} \cdots k_{q-2}^{\prime}\right)^{m^{2} / \beta-m}}{\left(k_{1} \cdots k_{q-1}\right)^{n\left(1-\alpha^{\prime} / \alpha\right)}} \frac{k_{q-1}^{\prime m^{2} / \beta-m}}{k_{q}^{n-\alpha^{\prime}}}+\sum^{q-1} \frac{\left(k_{1} \cdots k_{i}\right)^{n^{2} / \alpha-n}}{\left(k_{1}^{\prime} \cdots k_{i}^{\prime}\right)^{m\left(n+\beta-m-\alpha^{\prime} / \beta\right.}}\right. \\
& \left.+\sum \frac{\left(k_{1}^{\prime} \cdots k_{i-1}^{\prime}\right)^{m^{2} / \beta-m}}{\left(k_{1} \cdots k_{i}\right)^{n\left(1-\alpha^{\prime} / \alpha\right)}}\right\} .
\end{aligned}
$$

Dans tous les cas le premier terme à droite tend vers zéro et les deux séries sont convergentes lorsque $q \rightarrow \infty$, car $k_{i}^{\prime}=k_{i}^{k_{i}}, k_{i+1}=k_{i}^{\prime k_{i}^{\prime}}, \alpha^{\prime}<\alpha$ et $m+\alpha^{\prime}$ $<n+\beta$. Par conséquent, les moyennes $\left\{u_{q}\right\}, q=1,2, \ldots$, sont uniformément bornées par une valeur finie $c^{*}$. Par suite pour chaque $q$ il existe $\kappa_{q}$ tel que $U_{i n+\alpha^{\prime}}^{u}\left(P_{\kappa q}^{(q)}\right)<c^{*}$. On en déduit que la capacité d'ordre $m+\alpha^{\prime}$ de $F \times F^{\prime}$ est positive. En effet, si $C_{e}^{\left(m+\alpha^{\prime}\right)}\left(F \times F^{\prime}\right)=0$, il existerait d'après la proposition 10 une répartition $\mu_{0}$ sur $F \times F^{\prime}$ telle que $U_{m+\alpha^{\prime}}^{\mu_{0}}(P)=\infty$ en chaque point de $F \times F^{\prime}$. Puisque $P_{\kappa_{q}}^{(q)}$ vient arbitrairement voisin de $F \times F^{\prime}$ lorsque $q \rightarrow \infty$, $U_{m+\alpha}^{y_{0}}\left(P_{\kappa, y}^{(q)}\right)$ $\rightarrow \infty$ avec $q$, ce qui est absurde. Ainsi $C_{e}^{\left(m+\alpha^{\prime}\right)}\left(F \times F^{\prime}\right)>0$. 
Enfin, si $n+\beta \geqslant m+\alpha$ et $\alpha=n$, alors $\beta=m$; on peut prendre pour $F$ et $F^{\prime}$ un cube dans $E_{n}$ et un cube dans $E_{m}$ respectivement. $\operatorname{Si} \alpha<n$ et $\beta=m$, nous prenons un ensemble de dimension a dans $E_{n}$ pour $F$, par exemple un ensemble de Cantor généralisé choisi dans 4.4 , et un cube dans $E_{m}$ pour $F^{\prime}$. Alors la dimension de $F \times F^{\prime}$ est $m+\alpha$ d'après le théorème 6 . Ainsi le théorème est complètement démontré.

\section{BiBLIOGRAPHIE}

[1] N. Aronszajn and K. T. Smith: Functional spaces and functional completion, Ann. Inst. Fourier, 6 (1956), pp. 125-185.

[2] A. S. Besicovitch: Concentrated and rarefied sets of points, Acta Math., 62 (1933), pp. $289-300$.

[3] A. S. Besicovitch: On existence of subsets of finite measure of sets of infinite measure, Indag. Math., 14 (1952), pp. 339-344.

[4] A. S. Besicovitch and P. A. P. Moran: The measure of product and cylinder sets, J. Lond. Math. Soc., 20 (1945), pp. 110-120.

[5] N. Bogoliouboff et N. Kryloff : La théorie générale de la mesure dans son application à l'étude des systèmes dynamiques de la mécanique non linéaire, Ann. Math., 38 (1937), pp. 65-113.

[6] L. Carleson: On a class of meromorphic functions and its associated exceptional sets, Thèse, Uppsala (1950).

[ 7 ] G. Choquet: Theory of capacities, Ann. Inst. Fourier, 5 (1955), pp. 131-295.

[8] G. Choquet: Les noyaux réguliers en théorie du potentiel, C. R. Acad. Sci. Paris, 243 (1956), pp. 635-638.

[9] R. O. Davies: Subsets of finite measure in analytic sets, Indag. Math., 14 (1952), pp. $488-489$.

[10: J. Deny et P. Lelong: Étude des fonctions sousharmoniques dans un cylindre ou dans un cône, Bull. Soc. Math. France, 75 (1947), pp. 89-112.

[11] H. G. Eggleston: The Besicovitch dimension of cartesian product sets, Proc. Camb. Phil. Soc., 46 (1950), pp. 383-386.

[12] H. G. Eggleston: A correction to a paper on the dimension of cartesian product sets, ibid., 49 (1953), pp. 437-440.

[13] H. Federer: Some integralgeometric theorems, Trans. Amer. Math. Soc., 77 (1954), pp. 238-261.

[14] G. Freilich: On the measure of cartesian product sets, ibid., 69 (1950), pp. 232-275.

[15] G. Freilich: Two-dimensional measure in 3-space, Proc. Amer. Math. Soc., 6 (1955), pp. 631-633.

[16] F. Hausdorff : Dimension und äusseres Mass, Math. Ann., 79 (1919), pp. 157-179.

[17] S. Kametani : Positive definite integral quadratic forms and generalized potentials, Proc. Imp. Acad. Japan, 20 (1944), pp. 7-14.

[18] S. Kametani: On Hausdorff's measure and generalized capacities with some of their applications to the theory of functions, Jap. J. Math., 19 (1945), pp. 217-257.

[19] S. Kametani : Progrès recent de la théorie du potentiel, Mathématiques modernes I, Tokyo (1950), pp. 62-105, (en japonais). 
[20] S. Kametani : A note on a metric property of capacity, Natur. Sci. Rep. Ochanomizu Univ., 4 (1953), pp. 51-54.

[21] J. M. Marstrand: The dimension of Cartesian product sets, Proc. Camb. Phil. Soc., 50 (1954), pp. 198-202.

[22] P. A. P. Moran: Additive functions of intervals and Hausdorff measure, ibid., 42 (1946), pp. 15-23.

[23] P. A. P. Moran : On plane sets of fractional dimensions, Proc. Lond. Math. Soc., 51 (1949), pp. 415-423.

[24] M. Ohtsuka : Capacité d'ensembles de Cantor généralisés, Nagoya Math. J., 11 (1957), pp. 151-160.

[25] J. Randolph : On generalizations of length and area, Bull. Amer. Math. Soc., 42 (1936), pp. 268-274.

Institut de Mathematiques

Université de Nagoya 